\title{
Emerging Two-Dimensional Gauge Theories in Rydberg Configurable Arrays
}

\author{
Alessio Celi@ ${ }^{1,2,3, *}$ Benoît Vermersch $\odot,{ }^{1,2, \dagger}$ Oscar Viyuela, ${ }^{4,5}$ Hannes Pichler, ${ }^{5,6}$ Mikhail D. Lukin, ${ }^{5}$ and Peter Zoller ${ }^{1,2}$ \\ ${ }^{1}$ Center for Quantum Physics, Faculty of Mathematics, Computer Science and Physics, \\ University of Innsbruck, Innsbruck A-6020, Austria \\ ${ }^{2}$ Institute for Quantum Optics and Quantum Information, Austrian Academy of Sciences, \\ Innsbruck A-6020, Austria \\ ${ }^{3}$ Departament de Física, Universitat Autònoma de Barcelona, 08193 Bellaterra, Spain \\ ${ }^{4}$ Department of Physics, Massachusetts Institute of Technology, Cambridge, Massachusetts 02139, USA \\ ${ }^{5}$ Department of Physics, Harvard University, Cambridge, Massachusetts 02318, USA \\ ${ }^{6}$ ITAMP, Harvard-Smithsonian Center for Astrophysics, Cambridge, Massachusetts 02138, USA
}

(Received 25 July 2019; revised manuscript received 20 February 2020; accepted 20 April 2020; published 16 June 2020)

\begin{abstract}
Solving strongly coupled gauge theories in two or three spatial dimensions is of fundamental importance in several areas of physics ranging from high-energy physics to condensed matter. On a lattice, gauge invariance and gauge-invariant (plaquette) interactions involve (at least) four-body interactions that are challenging to realize. Here, we show that Rydberg atoms in configurable arrays realized in current tweezer experiments are the natural platform to realize scalable simulators of the Rokhsar-Kivelson Hamiltonian-a 2D U(1) lattice gauge theory that describes quantum dimer and spin-ice dynamics. Using an electromagnetic duality, we implement the plaquette interactions as Rabi oscillations subject to Rydberg blockade. Remarkably, we show that by controlling the atom arrangement in the array we can engineer anisotropic interactions and generalized blockade conditions for spins built of atom pairs. We describe how to prepare the resonating valence bond and the crystal phases of the Rokhsar-Kivelson Hamiltonian adiabatically and probe them and their quench dynamics by on-site measurements of their quantum correlations. We discuss the potential applications of our Rydberg simulator to lattice gauge theory and exotic spin models.
\end{abstract}

DOI: $10.1103 /$ PhysRevX.10.021057

\section{INTRODUCTION}

Atoms trapped in tweezer arrays and interacting via van der Waals interactions of laser-excited Rydberg states have recently emerged as one of the most promising platforms for quantum simulation of spin models. Unique features of Rydberg tweezer arrays include the flexibility of freely arranging atoms in any geometric structure in one, two, or three spatial dimensions [1-3]. In combination with strong, and potentially angular-dependent, Rydberg-Rydberg interactions, such arrays yield a versatile tool available to realize a wide variety of effective spin models, as demonstrated in recent experiments with Ising-type [4-6] and topological Su-Schrieffer-Heeger models [7] (for alternative

\footnotetext{
*Corresponding author. alessio.celi@uab.cat

Present address: Univ. Grenoble Alpes, CNRS, LPMMC, 38000 Grenoble, France.

Published by the American Physical Society under the terms of the Creative Commons Attribution 4.0 International license. Further distribution of this work must maintain attribution to the author(s) and the published article's title, journal citation, and DOI.
}

Subject Areas: Atomic and Molecular Physics,

Condensed Matter Physics realizations in optical lattices, see Refs. [8,9]). A key element of quantum many-body systems in Rydberg tweezer arrays is the Rydberg blockade mechanism [10]. Here, only single atomic Rydberg excitations within a given blockade radius $R_{c}$ are allowed, with double excitations strongly suppressed by large energy shifts from Rydberg van der Waals interactions. In this paper, we show that such an experimental setting provides a natural framework for implementing 2D U(1) lattice gauge models for spin 1/2 and, in particular, (a variant of) the RokhsarKivelson Hamiltonian [11]. Such a Hamiltonian corresponds to a paradigmatic model of quantum spin ice and quantum dimers [12]. Configurable 2D atomic tweezer arrays thus offer a unique opportunity to study RokhsarKivelson dynamics and phase diagrams, in particular, accessing and characterizing its resonating valence bond phase [13-15].

The implementation of lattice gauge theories in spatial dimensions larger than one is presently one of the key challenges in the ongoing development of quantum simulators. Recently, pioneering experiments have demonstrated the quantum simulation of 1D lattice gauge theories, including the 1D Schwinger model, as 1D quantum electrodynamics, with trapped ions $[16,17]$, and 
superconducting qubits [18]. Furthermore, recent experiments with 1D Rydberg chains [5] could be reinterpreted in terms of a truncated 1D Schwinger model [19] and string breaking. In contrast, lattice gauge theories in higher spatial dimensions are much harder to simulate. They are expected to display a plethora of novel physical phenomena which are absent in 1D, due to the interplay between electric and magnetic interactions, such as confined-deconfined phase transitions [20] and topological order [21]. A difficulty in implementing lattice gauge theories in higher spatial dimensions is that gauge invariance (Gauss law) and gauge-invariant magnetic interactions, plaquette terms, typically translate into four-body (or higher-order) interactions. This difficulty also applies when gauge field excitations are represented as finite dimensional, such as in lattice gauge spin models [22-25]. While recent proposals report significant advances in constructing gaugeinvariant terms in Kogut-Susskind-like [26] Hamiltonians from basic and natural building blocks, e.g., in cold atom systems [27-33], a laboratory implementation of 2D lattice gauge theories remains elusive (for a digital approach, see, e.g., Refs. [34-37]; for reviews, see Refs. [38-40]; for Floquet engineering and related progress with density-dependent gauge fields, see Refs. [41-45]). For instance, plaquette (ring-exchange) interactions have been experimentally demonstrated for disconnected plaquettes only [46].

In this work, we take a different route for achieving a natural implementation of 2D U(1) spin- $1 / 2$ models. The enabling insight is the existence of a dual formulation, where plaquette interactions are mapped into single-body terms with constraints. In the context of our Rydberg tweezer array, these correspond to Rabi couplings between atomic states which are subjected to generalized blockade conditions due to Rydberg interactions. Thus, we obtain a natural relation between the gauge theories and atomic systems with generalized blockade constraints, which provides a physical basis for the scalable [47] quantum simulation of lattice gauge theories in 2D.

The idea of exploiting dualities for quantum simulation of spin models and lattice gauge theories is not new; see, e.g., Ref. [49]. It is well known since Ref. [26] that in the magnetic basis the pure gauge Kogut-Susskind Hamiltonian simplifies (it is the same duality that relates $Z_{2}$ gauge theory like the toric code to the Ising model [50,51]) and allows one, e.g., to rewrite the Higgs-U(1) Hamiltonian [52] as an extended Bose-Hubbard model [53] (see also Refs. [54,55]). However, the duality we construct here for the pure gauge $\mathrm{U}(1)$ spin-1/2 models (quantum spin ice in the condensed matter language) has not been exploited for a quantum simulation previously.

The paper is organized as follows. In Sec. II, we introduce the Rokhsar-Kivelson Hamiltonian as a 2D $\mathrm{U}(1)$ spin- $1 / 2$ gauge theory and derive the dual Rokhsar-Kivelson (RK) Hamiltonian. First, after a brief tutorial on lattice gauge theories, we review the phase diagram of the RK Hamiltonian on the square lattice without background charges as known in its original basis (Sec. II A). Then, we define the duality transformation, describe its properties, and illustrate the phase diagram in terms of the observables of the dual spins on the full square lattice and on ladder geometries (Sec. II B). In Sec. III, we show how such dynamics can be naturally realized in 2D Rydberg arrays. First, we engineer 2D Ising models with tunable anisotropic interactions in decorated arrays, obtained by arranging orientable pairs of Rydberg atoms on 2D arrays (Sec. III A). The gauge theory emerges in such models for properly chosen arrangements (interactions) in the limit of small Rabi coupling (= transverse field), i.e., in a generalized blockade regime (Secs. III B and IIIC). We show that such Rydberg Rokhsar-Kivelson Hamiltonians host resonating valence bond phases, and we propose a step-by-step prescription to prepare and detect these phases in current experiments (Sec. III D). Finally, in Sec. IV, we summarize our results and discuss future steps and potential applications of our simulator based on controllable Rydberg arrays to gauge theories and beyond.

\section{RYDBERG GAUGE THEORIES: DUAL FORMULATION IN TERMS OF GENERALIZED BLOCKADES}

In this section, we show how to formulate a relevant $\mathrm{U}(1)$ gauge theory in terms of interactions that are natural for an atomic system. Let us start by introducing the gauge theory in simple terms. We focus on the situation in which the gauge field evolves in a background of static charges (possible extensions are discussed in the outlook).

\section{A. Spin gauge theories and the Rokhsar-Kivelson model}

The Hamiltonian of a gauge theory in two (or more) dimensions is constructed in terms of electric and magnetic interactions and of their coupling to charges. In continuous Abelian U(1) gauge theories relevant in high-energy physics like quantum electrodynamics (and similarly for non-Abelian gauge theories like quantum chromodynamics), the former are simply given by the square of the electric and magnetic fields $E_{\mu}$ and $B$, respectively, with $E_{\mu}=\partial_{t} A_{\mu}$ and $B=\partial_{x} A_{y}-\partial_{y} A_{x}$ defined (in the unitary gauge) through the vector potential $A_{\mu}$. Here, $t, x, y$ are the time and space coordinate in $2 \mathrm{D}$, and $\mu=x, y$. Gauge invariance, i.e., invariance of the Hamiltonian under local phase (symmetry) transformations of the charges, follows directly from the invariance of $E_{\mu}$ and $B$ under $A_{\mu} \rightarrow A_{\mu}+\partial_{\mu} \theta(x, y)$. The electric field is sourced by the charges through the Gauss law $\partial_{\mu} E_{\mu}=4 \pi Q$, where $Q$ is the charge density.

In gauge theories defined on the lattice [56], the charges occupy the sites $s=\left(x_{s}, y_{s}\right)$ of the lattice, while the 
electromagnetic field lives on its links $l$. The links are oriented and can be denoted by their starting site and their direction $\mu=x, y, l=(s, \mu)$. The electric interactions are defined directly in terms of the electric operator $\hat{E}_{s, \mu}$, a Hermitian operator of discrete spectrum acting on the links. For each link, one further defines a Wilson operator $\hat{U}_{s^{\prime}, \mu^{\prime}}$ as the lowering operator for the electric field: $\left[\hat{E}_{s, \mu}, \hat{U}_{s^{\prime}, \mu^{\prime}}\right]=$ $-\hat{U}_{s, \mu} \delta_{s, s^{\prime}} \delta_{\mu, \mu^{\prime}}$. The Wilson operator measures the phase acquired by a unit charge moved along the link $(s, \mu)$ of length $a$, i.e., $\hat{U}_{s, \mu} \sim e^{i a A_{\mu}(s)}$. The magnetic interactions are given by (oriented) products of these Wilson operators on the links around the plaquettes of the lattice. These operators are used to construct the Kogut-Susskind Hamiltonian [26]. In the limit of infinitely massive charges, such a Hamiltonian contains no dynamics for the charges and reads

$$
\begin{aligned}
H_{\mathrm{KS}}= & \sum_{s}\left[\frac{g^{2}}{2}\left(\hat{E}_{s, x}^{2}+\hat{E}_{s, y}^{2}\right)\right. \\
& \left.-\frac{1}{2 g^{2}}\left(\hat{U}_{s, x}^{\dagger} \hat{U}_{s+\hat{x}, y}^{\dagger} \hat{U}_{s+\hat{y}, x} \hat{U}_{s, y}+\text { H.c. }\right)\right],
\end{aligned}
$$

which reduces to the pure gauge $\mathrm{U}(1)$ Hamiltonian in the continuum, $H=\int\left(E^{2}+B^{2}\right)$, when the lattice spacing $a$ is sent to zero. The Hamiltonian (1) is gauge invariant, as it commutes with the lattice version of the Gauss law

$$
\begin{aligned}
& \left(\hat{E}_{s, x}+\hat{E}_{s, y}-\hat{E}_{s-\hat{x}, x}-\hat{E}_{s-\hat{y}, y}-\hat{Q}_{s}\right)|\Phi\rangle=0, \quad \forall s, \\
& \quad \Leftrightarrow|\Phi\rangle \in\{\text { physical states }\},
\end{aligned}
$$

that determines what states are physical for a given distribution of charges. Here, $\hat{Q}_{s}$ is the operator measuring the charge on the site $s$, and $|\Phi\rangle$ represents the state of the whole lattice, including both links and sites. The electric states, i.e., the eigenstates of the electric operators on the links, form a convenient basis for the link degrees of freedom. In particular, the physical states can be easily identified in this basis via Eq. (2).

Since the electric field is unbounded, the number of electric states on each link is, in principle, infinite. However, it is possible to truncate it to a maximal value and define consistently $\mathrm{U}(1)$ [and $\mathrm{SU}(\mathrm{N})[57,58]$ ] lattice gauge theories with finite local Hilbert spaces (at the price that the Wilson operator is no longer unitary). The simplest $\mathrm{U}(1)$ gauge theories in 2D, known as gauge magnets, link models, and Ising gauge theories [22-25], are obtained by considering just two electric states per link; see Fig. 1. The electric operator reduces to $\hat{E}_{s, \mu} \rightarrow \hat{S}_{s, \mu}^{z}$ and the Wilson operator to $\hat{U}_{s, \mu} \rightarrow \hat{S}_{s, \mu}^{-}$, with $\hat{S}^{ \pm}=\hat{S}^{x} \pm i \hat{S}^{y}$, and the physical configurations and their dynamics follow from Eqs. (2) and (1).

We can represent the physical configurations in the (electric) $\hat{S}^{z}$ basis by coloring in red the links in $|\uparrow\rangle$

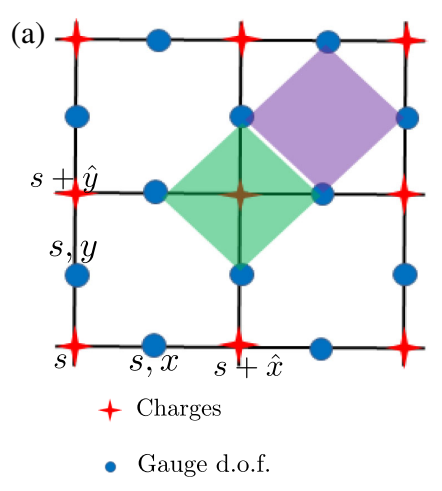

(b)
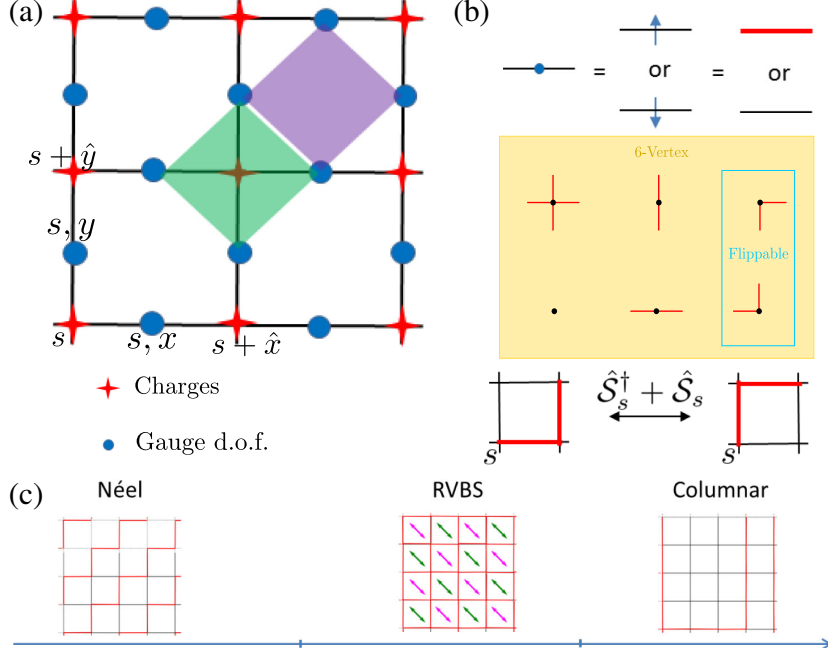

RVBS

Columnar

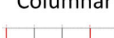

FIG. 1. Spin gauge theories and the Rokhsar-Kivelson model. (a) Spin-1/2 lattice gauge theories are the simplest gauge theories with a continuous gauge symmetry group. The gauge degrees of freedom are spins $1 / 2$ that live on the oriented links of the lattice, labeled as $s, \mu$, where $s$ is the starting site and $\mu$ is the direction, e.g., $x$ or $y$ in a square lattice. They display the main features of gauge theories in $D>1$ : (i) the Gauss law that determines the allowed spin configurations on the links in terms of the charges and (ii) the magnetic interactions acting on the links around a plaquette. On a square lattice, these operators correspond to both four-body spin operators indicated by the green and violet rhombi, respectively. (b) In a system without charges, we depict the effect of the Gauss law by coloring in red the links in $|\uparrow\rangle$ and not coloring the ones in $|\downarrow\rangle$ that represent the electric states. Physical states are a superposition of endless red strings going up and right [see (c) and Fig. 2]. Such electric configurations correspond to position states, and $\hat{\mathcal{S}}_{s}^{\dagger}+\hat{\mathcal{S}}_{s}$ acts as a kinetic term on them. (c) The square of the plaquette operator $\left(\hat{\mathcal{S}}_{s}^{\dagger}+\hat{\mathcal{S}}_{s}\right)^{2}$ is diagonal in the electric basis and plays the role of a potential term that counts flippable plaquettes. The competition of the kinetic and potential terms in the Rokhsar-Kivelson Hamiltonian (4) gives rise to a rich phase diagram, with the resonating valence bond solid (RVBS) phase separating two crystal phases, the Néel and the columnar phases (see the description in the main text). The resonant plaquettes are depicted by diagonal double arrows. The alternated green and pink arrows reflect that the resonant plaquettes are correlated within the same sublattice and anticorrelated with the ones in the opposite sublattice.

and not coloring the ones in $|\downarrow\rangle$. We use such notation in Fig. 1(b) to illustrate the six configurations out of 16 allowed around a site without charges. All the physical configurations of the plane are obtained by assembling the local building blocks satisfying Eq. (2).

Since $E^{2}$ is trivial $\left[\left(\hat{S}^{z}\right)^{2}=1\right]$ in the retained states [59], the Kogut-Susskind Hamiltonian (1) specialized to the truncated theory contains only magnetic interactions $H_{\mathrm{KS}} \rightarrow-\sum_{s} \hat{\mathcal{S}}_{s}+\hat{\mathcal{S}}_{s}^{\dagger}$ with

$$
\hat{\mathcal{S}}_{s}+\hat{\mathcal{S}}_{s}^{\dagger}=\hat{S}_{s, x}^{-} \hat{S}_{s+\hat{x}, y}^{-} \hat{S}_{s+\hat{y}, x}^{+} \hat{S}_{s, y}^{+}+\text {H.c. },
$$


where we label the plaquette by its lower left site. As shown in Fig. 1(b), $\hat{\mathcal{S}}_{s}+\hat{\mathcal{S}}_{s}^{\dagger}$ interchanges two electric configurations of a plaquette, while it annihilates the remaining (14) ones. In the following, we refer to these two configurations as flippable and to $\hat{\mathcal{S}}_{s}+\hat{\mathcal{S}}_{s}^{\dagger}$ as the plaquette operator. Notice that neighbor plaquette operators are not commuting: Such a property is a consequence of the electric field truncation and has important implications on the dynamics. In fact, we can interpret the truncation as the effect of a modified electric term; see the Appendix B. Thus, the usual dynamical competition between electric and magnetic interactions is retained and transformed from a soft into a hard constraint.

The Hamiltonian considered so far is not the most general one compatible with gauge invariance, i.e., commuting with Eq. (2), that one can construct. If we regard the electric basis as a position basis, we can interpret the sum of plaquette operators as a kinetic term that acts on the electric configurations by interchanging them. We can thus add potential terms that are diagonal in the electric basis and weight the different configurations. The resulting lattice models have a rich phase diagram and are of direct interest in condensed matter even without taking the continuum limit. A relevant Hamiltonian in this class is the RK model [11]

$$
H_{\mathrm{RK}}=-J \sum_{s}\left[\left(\hat{\mathcal{S}}_{s}+\hat{\mathcal{S}}_{s}^{\dagger}\right)-\lambda\left(\hat{\mathcal{S}}_{s}+\hat{\mathcal{S}}_{s}^{\dagger}\right)^{2}\right],
$$

where the potential term is given by the square of the plaquette operator. Here, the potential term simply counts the number of flippable plaquettes, and, therefore, $\lambda$ plays the role of a chemical potential for the flippable electric configurations. The RK Hamiltonian was originally proposed as a simple model of close-packed quantum dimers that could host short-range resonating valence bond [13-15] insulating states. Quantum dimers are an effective description of valence bonds, the singlets of valence electrons in a quantum antiferromagnet. Originally, it was thought that in the presence of doping [which implies the addition of kinetic terms for dimers in Eq. (4)] the resonating valence bond state would become a superconductor due to the condensation of the vacancies (holon), such to provide a mechanism for high$T_{c}$ superconductivity [61] in cuprates. Nowadays, it is still expected that the pseudogap phases at moderate hole doping are resonating valence bond phases although of a more complex nature [62], and the properties of the latter are well understood in terms of emerging gauge theories [63], as first realized in Refs. $[64,65]$. Dimer configurations are equivalent to electric configurations in the presence of a staggered distribution of static charges \pm 1 , and their dynamics is described by Eq. (4); see Appendix A for further details.

\section{Phase diagram of the RK model}

The RK Hamiltonian has a rich phase diagram in any lattice geometry and charge distribution. At $\lambda=1$, known as the RK point, the Hamiltonian (4) becomes a sum of projectors and is semipositive definite by construction [11]. The equal superposition of all the allowed configurations is the exact, zero energy ground state of $H_{\mathrm{RK}}(\lambda=1)$. This state is a prototype of a quantum spin liquid [66]. In the square lattice and with no charges [see Fig. 1(c)], the RK point separates a columnar phase for $\lambda>1$, from a resonating valence bond solid (RVBS) phase $[67,68]$ with quasi-long-range order that extends from $\lambda_{c}<\lambda<1$. For $\lambda=\lambda_{c} \sim-0.3$, there is a weakly-first-order phase transition $[69,70]$ to a Néel phase. Both the Néel and the RVBS are examples of order by disorder [71], where quantum fluctuations resolve the classical degeneracy and select a unique ground state. The RVBS is also a crystal order phase but richer: Similarly to the plaquette phase [72] in the quantum dimer model, it preserves the point symmetry of the lattice and can be interpreted as the oscillation between the two Néel states. Its correlation pattern [see Fig. 1(c)] spontaneously breaks translational invariance while preserving charge conjugation [73].

On frustrated lattices like the triangular and kagome ones, the corresponding RK Hamiltonians for quantum dimers [74] are expected to display a true spin-liquid phase around the RK point, $\lambda \lesssim 1$ [75-77], while the overall structure of the phase diagram is lattice dependent; see, e.g., Fig. 17.8 in Ref. [12].

The multibody interactions in Hamiltonians like the RK model (4) make experimental observations challenging, both in condensed matter systems and in synthetic quantum matter. The relevant Hamiltonian terms can, in principle, be obtained as low-energy limits of antiferromagnets, for instance, in the 2D pyrochlore lattice in the Ising limit [76]. There, the dominating Ising spin-spin interactions impose the Gauss law on the low-energy manifold, and the plaquette interactions emerge in perturbation theory through ring exchange $[29,78]$. Alternatively, one can engineer both Gauss law and plaquette interactions by adopting a digital approach $[34,79]$ based on Rydberg gates [80]. In both cases, the suppression of the energy scale or complexity of the digital procedures makes the realization of 2D gauge theories in experiments extremely hard.

\section{B. Dual formulation of the RK model}

We show here that it is possible to reformulate spin gauge theories such that the plaquette interactions acquire a simpler form. In particular, we find a dual formulation where the multibody interactions have a natural realization in atomic arrays with Rydberg interactions. For the sake of concreteness, let us focus on the RK model on the square lattice without charges (and fix the boundary conditions compatible with the Néel state); see Figs. 1(c) and 2. For the dual formulation, we consider a spin- $1 / 2$ system associated with each plaquette of the square lattice. The physical states of the original gauge theory are related to states of the plaquette spins via the operator identification 


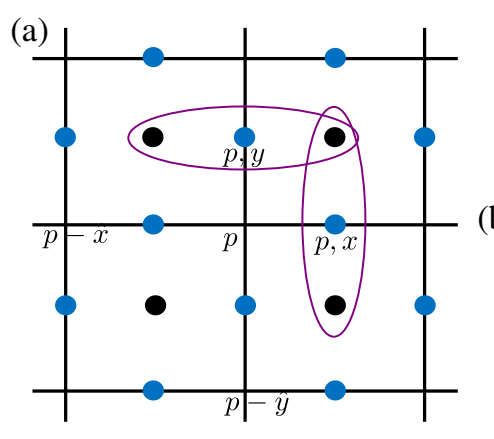

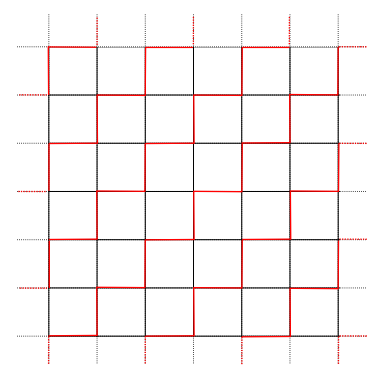

(b)

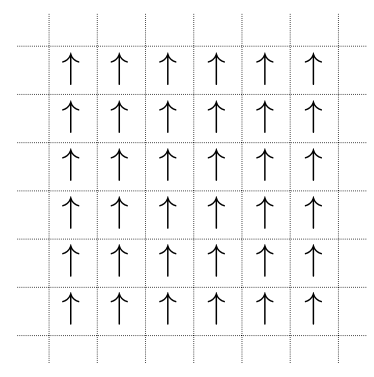

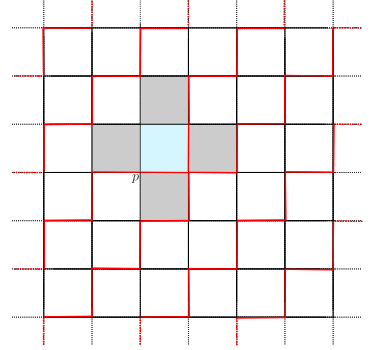

(c)

$\left(\hat{\mathcal{S}}_{p}+\hat{\mathcal{S}}_{p}^{\dagger}\right)|\Omega\rangle$

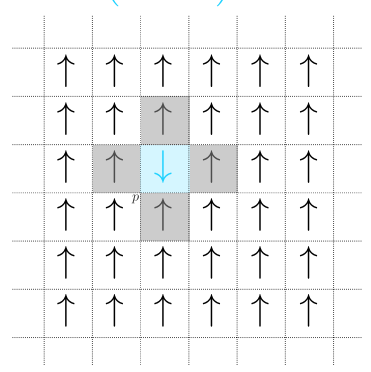

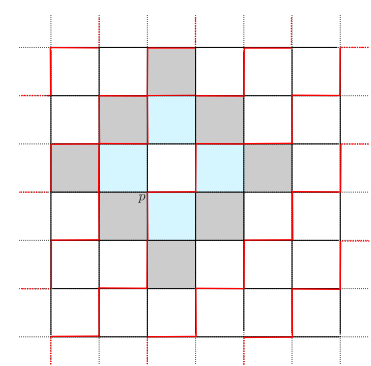

(d)
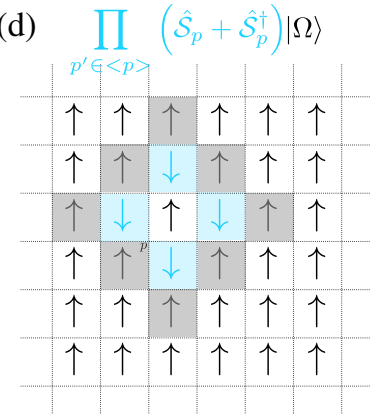

FIG. 2. Graphical illustration of the duality. (a) Definition of the link spins (blue dots) in terms of the dual plaquette spins (black dots), as in Eq. (5). The dual spins live in the dual lattice formed by the centers of the plaquettes. The value of the link spin is determined by the plaquette spins of the two plaquettes sharing the link, as indicated by the purple ellipses for the links $p, x$ and $p, y$. The orientation of the plaquette spins is chosen such that the fully flippable state $|\Omega\rangle$ is mapped in the dual basis into the ferromagnetic state (b). (b)-(d) We represent the configurations that are relevant to understand the generalized blockade condition simultaneously in the original (above) and the dual (below) basis. In the former, we represent the link spins with the color convention in Fig. 1. In the latter, we represent the dual plaquette spins as arrows placed in the center of the corresponding plaquettes. (b) The reference state $|\Omega\rangle$ : We identify it with the ferromagnetic state with all plaquette spins up. (c) After flipping one plaquette of $|\Omega\rangle$, all the neighboring plaquettes are blocked. (d) After flipping all its neighboring plaquettes, the plaquette $p$ is flippable.

$$
\begin{aligned}
& \hat{S}_{p, x}^{z} \rightarrow-2(-1)^{p} \hat{S}_{p}^{z} \hat{S}_{p-\hat{y}}^{z}, \\
& \hat{S}_{p, y}^{z} \rightarrow 2(-1)^{p} \hat{S}_{p}^{z} \hat{S}_{p-\hat{x}}^{z},
\end{aligned}
$$

where the $\hat{S}_{p}^{z}$ acts on the spin $1 / 2$ associated to the plaquette $p=\left(x_{p}, y_{p}\right)$ and $(-1)^{p}=(-1)^{x_{p}+y_{p}}$ distinguishes even $(+)$ and odd (-) plaquettes. Equation (5) defines a one-to-one mapping between Hilbert spaces that is well defined up to an overall $Z_{2}$ identification of the plaquette spins for periodic boundary conditions. For open lattices, the $Z_{2}$ degeneracy can be removed, for instance, by choosing all the dual spins up on the boundary. Note that the transformation (5) maps the Néel state into the ferromagnetic states, $\left.|\Omega\rangle=\prod_{p \in \text { even }}\left|\ulcorner\rangle_{p} \rightarrow \prod_{p}\right| \uparrow\right\rangle_{p}$; see Fig. 2(a).

This mapping is appealing, because the Hamiltonian (4) is particularly simple in this dual formulation. Since the map (5) is quasilocal, it maps local operators into local operators. In particular, as detailed in Appendix C, the plaquette operator can be written as

$$
\hat{\mathcal{S}}_{p}+\hat{\mathcal{S}}_{p}^{\dagger} \rightarrow\left(P_{p}^{\uparrow \uparrow \uparrow \uparrow}+P_{p}^{\downarrow \downarrow \downarrow \downarrow}\right) 2 \hat{S}_{p}^{x} .
$$

Here, $P_{p}^{\uparrow \uparrow \uparrow \uparrow} \equiv \prod_{p^{\prime} \in\langle p\rangle}\left(\frac{1}{2}+\hat{S}_{p^{\prime}}^{z}\right)$ denotes the projector onto states where all spins neighboring to $p$ are in the state $|\uparrow\rangle$ (and analogously $P_{p}^{\downarrow \downarrow \downarrow \downarrow}$ ). Even though the plaquette operator is a multispin operator also in this dual representation, it has a simple form: It flips the spin of the associated plaquette, conditional on the state of its nearest neighbors. In particular, a single plaquette spin is flipped by the plaquette operator only if all four spins on the neighboring plaquettes point in the $\uparrow$ direction or if all point in the $\downarrow$ direction. The (dual) RK Hamiltonian is simply given by a sum of such terms:

$$
H_{\mathrm{RK}}^{*}=-J \sum_{p}\left(P_{p}^{\uparrow \uparrow \uparrow \uparrow}+P_{p}^{\downarrow \downarrow \downarrow \downarrow}\right)\left(2 \hat{S}_{p}^{x}-\lambda\right) .
$$

Note that the map (5) and the Hamiltonian (7) are the quantum version of the height formalism [81-83] applied to the RK model without charges; see Appendix C. The same map can be derived also for other charge sectors and geometries [84], including frustrated lattices [85] (see Sec. IV for further discussion).

To illustrate this duality further, let us consider the repeated action of the plaquette operator on the fully flippable state $|\Omega\rangle$. Once the first plaquette operator $\left(\hat{\mathcal{S}}_{p}+\right.$ $\left.\hat{\mathcal{S}}_{p}^{\dagger}\right)$ is applied, all spin on plaquettes $p^{\prime}$ neighboring $p$ can no longer be flipped. This result reflects the gauge constraint (and the electric-field truncation) and manifests in 
(a)

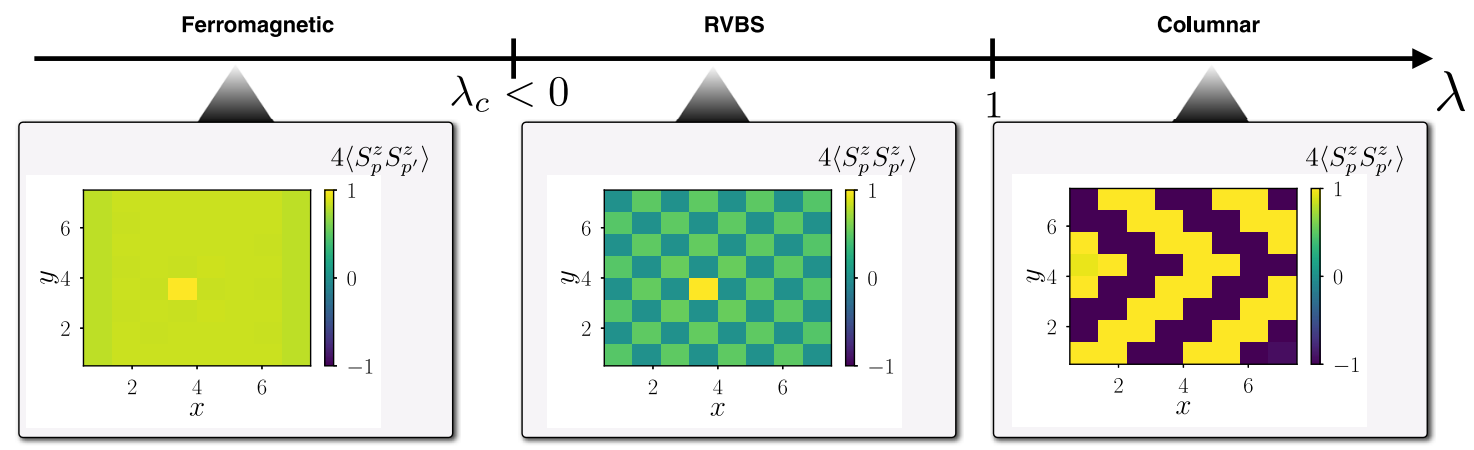

(b)

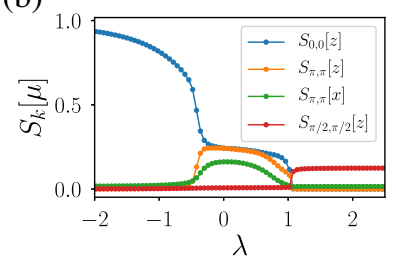

(c)

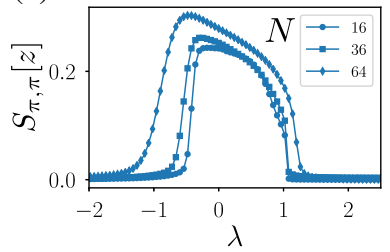

(d)

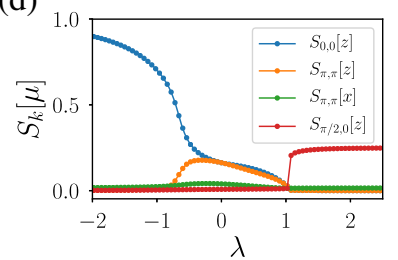

(e)

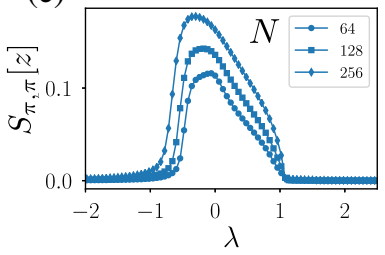

FIG. 3. Dual RK model and its phases. (a) Phase diagram of the 2D RK model on the square lattice without background charges. In the boxes, we plot the spin-spin correlations $\left\langle S_{p}^{\mu} S_{p^{\prime}}^{\mu}\right\rangle$ obtained via DMRG [87] on square lattices $N_{x} \times N_{y}=8 \times 8$ and for three values of $\lambda=-1,0,2$ that belong to the ferromagnetic (Néel), RVBS, and columnar phases, respectively. The correlations are represented with respect to the center, $p=(4,4)$, for varying $p^{\prime}$. Note that in the $\lambda \rightarrow \infty$ limit there are multiple degenerate unflippable states. The particular configuration shown here in the right panel corresponds to one of them. (b), (c) Structure factors $S_{k}[\mu]=$ $4 /\left(N_{x} N_{y}\right)^{2} \sum_{p, p^{\prime}} \exp \left[i\left(p-p^{\prime}\right) k\right]\left\langle\hat{S}_{p}^{\mu} \hat{S}_{p^{\prime}}^{\mu}\right\rangle, \mu=x, z$ for $N_{x} \times N_{y}=8 \times 8$ [(b)] and for different system sizes $N=N_{x} N_{y}=N_{x}^{2}[(\mathrm{c})]$. The raising of the structure factors $S_{(\pi, \pi)}[x, z]$ identifies the RVBS phase. In particular, $S_{(\pi, \pi)}[z]$ equals $S_{(0,0)}[z]$ in the RVBS region. (d),(e) Structure factors on the periodic ladder of $N_{x} \times 2$ sizes, without background charges, with $N_{x}=32[(\mathrm{~d})]$ and $N_{x}=32$, $64,128[(\mathrm{e})]$.

the annihilation of the state by the corresponding plaquette operator, $\left(\hat{\mathcal{S}}_{p^{\prime}}+\hat{\mathcal{S}}_{p^{\prime}}^{\dagger}\right)\left(\hat{\mathcal{S}}_{p}+\hat{\mathcal{S}}_{p}^{\dagger}\right)|\Omega\rangle=0$ [see Fig. 2(c)]. In the context of Rydberg physics, such conditional or constraint dynamics is well known as the blockade effect. However, in contrast to the standard blockade mechanism, where a spin can be flipped only if all its neighbors are in a single, specific configuration, here two distinct configurations of its neighbors allow a spin to flip. We thus refer to the present situation as the generalized blockade condition. Specifically, if all of the remaining three spins on plaquettes neighboring to $p^{\prime}$ are flipped, then the spin $p^{\prime}$ becomes flippable again; see Fig. 2(d). It is easy to see that one can, in fact, span all physical states - the states that satisfy the gauge constraint-(and no other states) by repeated application of plaquette operators.

\section{Phase diagram of the RK model in the dual formulation}

We conclude by discussing the phase diagram and, in particular, the RVBS phase in the dual picture (7); see Fig. 3. There, we show also the corresponding structure factors $\quad S_{k}[\mu]=\sum_{p, p^{\prime}} 4 /\left(N_{x} N_{y}\right)^{2} \exp \left[i\left(p-p^{\prime}\right) k\right]\left\langle\hat{S}_{p}^{\mu} \hat{S}_{p^{\prime}}^{\mu}\right\rangle$ calculated by the density matrix renormalization group (DMRG) using ITensor library [87] on square lattices $N_{x} \times$ $N_{y}$ up to $8 \times 8$. We ensure that the energy is minimized only on accessible states from $|\Omega\rangle$, for which the map is defined (see Appendix E). The phase for $\lambda<\lambda_{c}$ is ferromagnetically ordered in each sublattice as expected for the fully flippable phase. The boundary conditions compatible with $|\Omega\rangle$ select the ferromagnetic order in both sublattices as evidenced by a large value of $S_{k}[z]$ for $k=(0,0)$, the blue curve in Fig. 3(b), which is the dominant contribution to spin-spin correlations. The RVBS for $\lambda_{c}<\lambda<1$ is correlated along both $z$ and $x$ within the two sublattices only. Such a structure is in agreement with RVBS being even under charge conjugation, and it is signaled by both the equality of the $S_{k}[z]$ for $k=(0,0)$ and $k=(\pi, \pi)$ (in orange) and the raising of the $S_{k}[x]$ (in green); see Figs. 3(b) and 3(c). The "unflippable" phase (it is reachable from $|\Omega\rangle$ only through the boundary where the residual flippable plaquettes appear as defects) for $\lambda>1$ displays a characteristic strip order with the plaquette spins aligning in the $z$ direction along the diagonals of the lattice, with an associated periodicity four captured by $S_{k}[z]$ for $k=(\pi / 2, \pi / 2)$. In Fig. 3(a), we show one of the possible configurations with two flippable plaquettes (defects) on the boundary. For $\lambda \rightarrow \infty$, we have as many exactly degenerate configurations as allowed positions for the defects. The degeneracy is only approximated at finite $\lambda>1$ (the degeneracy is resolved): In Fig. 3(a), we show the resulting configuration. 


\section{Special cases: Ladder geometries}

The dual RK Hamiltonian (7) is reminiscent of Rydberg physics and of the PXP model [5,88,89], which can be obtained from Eq. (7) by replacing the projector $P_{p}^{\uparrow \ldots \uparrow}+$ $P_{p}^{\downarrow \ldots \downarrow}$ with the simpler blockade condition $P_{p}^{\uparrow \ldots \uparrow}$ ( or $P_{p}^{\downarrow \ldots \downarrow}$ ). It is noticed in Ref. [90] that the quantum dimer model on a ladder can be mapped into a $P X P$-like model on a chain. Indeed, if we restrict the Hamiltonian (7) to a chain immersed in the background $|\Omega\rangle$, only the blockade term $P_{p}^{\uparrow \uparrow}=\left(\frac{1}{2}+\hat{S}_{p-\hat{x}}\right)\left(\frac{1}{2}+\hat{S}_{p+\hat{x}}\right)$ survives. Precisely, the resulting Hamiltonian coincides with Eq. (3) in Ref. [88] for $\omega=J$ and $U=-2 V=-2 \lambda J$. It is not charge conjugation invariant, and the corresponding phase diagram for varying $\lambda$ does not show any analog of the RVBS phase, which is even under charge conjugation; see Fig. 6 in Appendix F.

Interestingly, the minimal geometry that preserves such symmetry is a periodic ladder, i.e., a cylinder with a circumference of two lattice sites. Since the dual lattice of a periodic ladder is a periodic ladder, $p+\hat{y}=p-\hat{y}$, the dual RK Hamiltonian takes the simple form

$$
H_{\mathrm{RK}}^{* p l}=-J \sum_{p}\left(P_{p}^{\uparrow \uparrow \uparrow}+P_{p}^{\downarrow \downarrow \downarrow}\right)\left(2 \hat{S}_{p}^{x}-\lambda\right),
$$

where we restrict $p$ to run over the sites of a square ladder, $p=\left(x_{p}, y_{p}\right)$ with $y_{p}=[0,1]$, such that $P_{x, y}^{\uparrow \uparrow \uparrow(\downarrow \downarrow \downarrow)}=$ $\left[\frac{1}{2}+(-) \hat{S}_{x-1, y}^{z}\right]\left[\frac{1}{2}+(-) \hat{S}_{x, y+1}^{z}\right]\left[\frac{1}{2}+(-) \hat{S}_{x+1, y}^{z}\right]$. As shown in Figs. 3(d) and 3(e), the phase diagram of Eq. (8) has the same structure as the one of the 2D RK model [Figs. 3(b) and 3(c)]. In particular, the RVBS phase is signaled by the equality of the structure factors $S_{k}[z]$ for $k=(0,0)$ and $k=(\pi, \pi)$.

\section{RYDBERG GAUGE THEORIES: RK HAMILTONIAN FROM DECORATED RYDBERG ARRAYS}

In this section, we discuss the implementation of the dual RK Hamiltonian (7) (and of analogous expression for other lattices) with Rydberg atoms. Our starting point is a $2 \mathrm{D}$ array of atoms with tunable geometry, driven between their ground $|g\rangle$ and Rydberg $|r\rangle$ states, respectively, with Rabi frequency $\Omega$ and detuning $\Delta(\hbar=1)$ :

$$
\begin{aligned}
H_{\mathrm{Ryd}}= & \sum_{I}\left[-\Omega\left(|r\rangle_{I}\langle g|+| g\rangle_{I}\langle r|\right)+\Delta n_{I}\right. \\
& \left.+\frac{1}{2} \sum_{I^{\prime} \neq I} \frac{C_{6}}{\left|I-I^{\prime}\right|^{6}} n_{I^{\prime}} n_{I^{\prime}}\right],
\end{aligned}
$$

where $n_{I}=|r\rangle_{I}\langle r|$ and $C_{6}$ is the van der Waals potential energy at unit distance. We arrange the atoms by pairs such to realize composite spins $1 / 2$.
We first show in Sec. III A that under suitable conditions $H_{\text {Ryd }}$ becomes equivalent to an anisotropic Ising model for the atom pairs with interactions and detuning determined by the pair arrangement. Then, in Secs. III B and III C, we achieve the generalized blockade from such a spin model by properly choosing the pair arrangement in a similar fashion as for ordinary blockade in Rydberg chains [5].

\section{A. Tunable Ising models from decorated Rydberg arrays}

We consider an array of atom pairs (see Fig. 4) such that the position of each atom $I$ is given in terms of the pair position $p$ and atom displacement in the pair $\eta, I=p+\sigma \eta$, $\sigma= \pm \frac{1}{2}$. We take $|\eta|$ sufficiently small (i.e., the pairs sufficiently far apart) such that Rydberg interactions between the atoms in the pairs is the dominating interaction, $\left(C_{6} /|\eta|^{6}\right) \gg \Omega,\left(C_{6} /\left|I(p)-I\left(p^{\prime}\right)\right|^{6}\right), p^{\prime} \neq p$. For a sufficiently blue-detuned driving field $(\Delta<0$ such that $\Omega \ll-\Delta$ ), the low-energy sector consists of configurations where exactly one atom per pair is in the Rydberg state and the other atom is in the internal ground state. Thus, each pair forms an effective pseudo spin-1/2 system, with the identification [see the inset in Fig. 4(a)]

$$
\hat{S}_{p}^{z}|g\rangle_{p-\sigma \eta}|r\rangle_{p+\sigma \eta}=\sigma|g\rangle_{p-\sigma \eta}|r\rangle_{p+\sigma \eta} .
$$

The effective Hamiltonian (9) in this low-energy limit becomes

$$
H_{\mathrm{Ryd}} \approx-2 J \sum_{p} \hat{S}_{p}^{x}+\sum_{k} V^{(k)},
$$

where $J$ is the effective Rabi frequency, $J=\Omega^{2}((1 / \Delta)+$ $\left.\left(|\eta|^{6} / C_{6}-\Delta|\eta|^{6}\right)\right)$, and $V^{(k)}$ is the van der Waals interactions between Rydberg atoms in $k$-neighbor pairs. Disregarding boundary and constant terms, $V^{(k)}$ can be written as Ising interactions between the $k$ spins plus a local term (such a term appears because the distance between the atoms in the Rydberg state in the pair-pair configurations $\uparrow_{p} \downarrow_{p^{\prime}}$ and $\downarrow_{p} \uparrow_{p^{\prime}}$ can differ):

$V^{(k)}=\sum_{p} \hat{S}_{p}^{z} \sum_{p^{\prime}=\langle p\rangle_{k}}\left[A\left(p-p^{\prime}, \eta\right) \hat{S}_{p^{\prime}}^{z}+B\left(p-p^{\prime}, \eta\right)\right]$,

where $\langle p\rangle_{k}$ denotes the $k$ neighbors of $p$. The coefficients $A$ and $B$ are determined by the geometry of the array: They are symmetric and antisymmetric functions of the pair separation and of the atom displacement in the pairs $\eta$ :

$$
\begin{gathered}
A(p, \eta)=C_{6}\left(\frac{2}{|p|^{6}}-\frac{1}{|p+\eta|^{6}}-\frac{1}{|p-\eta|^{6}}\right) \\
B(p, \eta)=C_{6}\left(\frac{1}{|p+\eta|^{6}}-\frac{1}{|p-\eta|^{6}}\right) .
\end{gathered}
$$




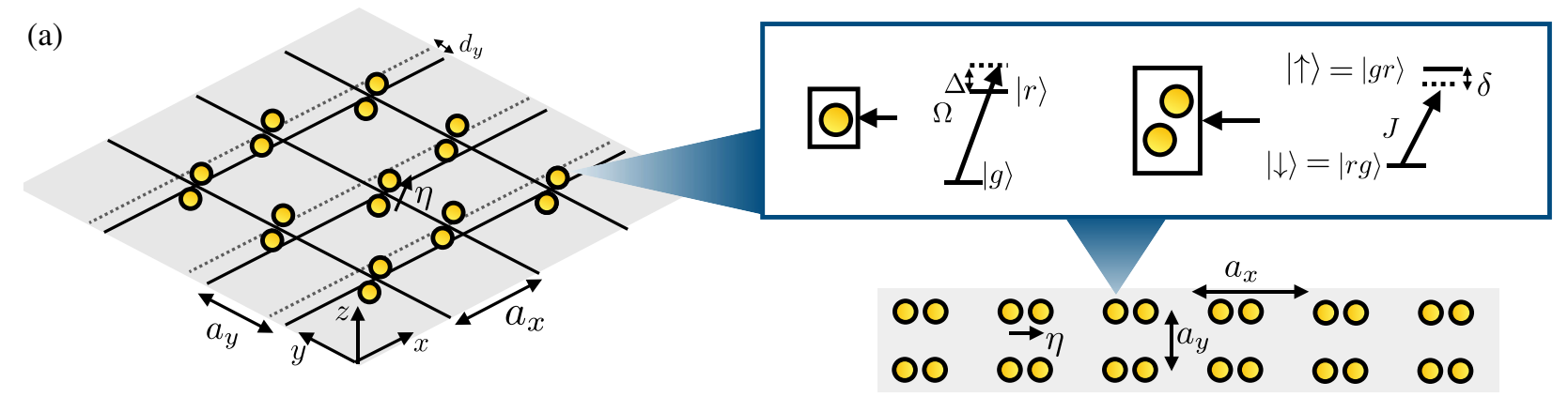

(b)

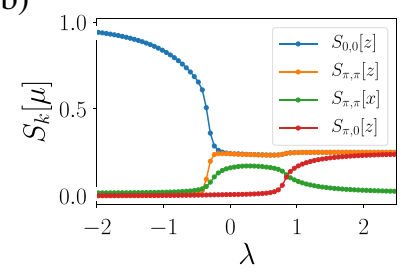

(c)

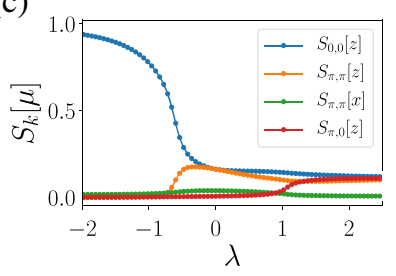

(d)

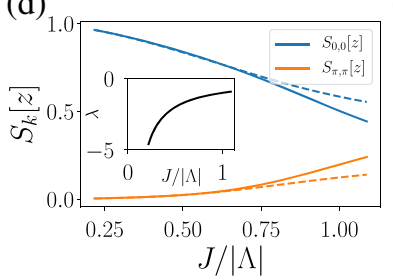

(e)

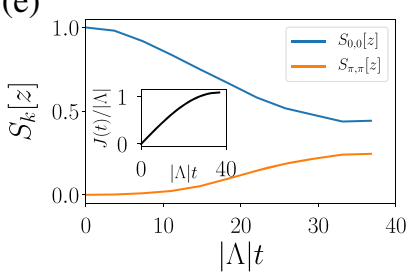

FIG. 4. RVBS in decorated Rydberg arrays. (a) Implementation of the RK Hamiltonian in decorated Rydberg arrays: Oriented pairs of close-by atoms with blue-detuned Rabi oscillations between the ground $|g\rangle$ and Rydberg $|r\rangle$ states are equivalent to oscillating spins $1 / 2$ (see inset) with Ising interactions between them. By controlling the pair orientation relative to the lattice and the lattice geometry, we can engineer the spin-spin interactions such to achieve the generalized blockade conditions and realize the RK Hamiltonian in the dual formulation with a modified RK potential. We name such Hamiltonians as Rydberg-RK Hamiltonians. On the left we show the square lattice construction and on the bottom right the implementation for a ladder. The same principle applies to a generic 2D lattice. (b),(c) The phase diagrams of the Rydberg RK Hamiltonian on the square lattice (18) $[N=8 \times 8$, (b)] and on the (periodic) ladder (15) $[N=32 \times 2,(\mathrm{c})]$ as a function of $\lambda$, calculated via DMRG. The two phase diagrams differ qualitatively from the ones of the original RK Hamiltonians shown in Figs. 3(b) and 3(c), respectively, for $\lambda \gtrsim 1$. In particular, the ferromagnetic and the RVBS phases remain, while the columnar phase is substituted by a "glassy" phase. (d) In order to compare the phase diagrams of the effective RK Hamiltonian with the full spin description of the decorated Rydberg array, we compare the spin-spin correlations on a $6 \times 2$ decorated ladder, for $|\eta|=0.38 a_{x}$ and periodic boundary conditions, via exact diagonalization. The structure factors for the ground state of the effective RK Hamiltonian and the corresponding eigenstates of the Rydberg array Hamiltonian (up to next-nearest-neighbor interactions) are shown, respectively, as dashed and solid lines. We included a small detuning $\delta=0.1 C_{6} / a_{x}^{6} \approx 0.11|\Lambda|$ within the atoms of each pair to select a specific ferromagnetic state as a reference. (e) Adiabatic preparation of the RVBS phase for the same parameters as (d). By smoothly ramping up the Rabi frequency $J$ (inset), we can enter the RVBS phase within the validity of generalized blockade condition, $J \ll G$.

Below, we show how to exploit their tunability for achieving the dual RK Hamiltonian for various lattice geometries. In such a construction, the pair-pair interactions $V^{(k)}$ for $k>2$ are negligible, and we consider them to be zero.

\section{B. RVBS phase on a decorated Rydberg ladder}

As a preliminary exercise, we consider the implementation of the RK Hamiltonian on a periodic ladder (8). Here, we parametrize the position of the pairs on the ladder as $p=\left(a_{x} x_{p}, a_{y} y_{p}\right)$, where $a_{x}$ and $a_{y}$ are the lattice spacing along the legs and the length of the rungs, respectively; see Fig. 4(a). For simplicity, we can express all lengths in units of $a_{x}, a_{x}=1$.

Let us focus on the projector appearing in Eq. (8). We can obtain it dynamically from the nearest-neighbor (NN) pair interactions $V^{(k=1)}$ in Eq. (12) if we require that $\sum_{p^{\prime}=\langle p\rangle_{1}} A\left(p-p^{\prime}, \eta\right)=0=\sum_{p^{\prime}=\langle p\rangle_{1}} B\left(p-p^{\prime}, \eta\right)$. By orienting the pairs along $x, \eta=|\eta| \hat{x}$, the constant term $B$ cancels out. The requirement on the NN Ising couplings reduces to $2 A(\hat{x}, \eta)=-A\left(a_{y} \hat{y}, \eta\right) \equiv-2 G(|\eta|)$, which fixes the lattice spacing $a_{y}$ (more precisely, the ratio $\left.a_{y} / a_{x}\right)$ in terms of $|\eta|$. With this choice, the Hamiltonian (11) of a decorated Rydberg ladder becomes

$$
\begin{aligned}
H_{\mathrm{Ryd}} & =V^{(2)}-\sum_{p}\left[2 J \hat{S}_{p}^{x}+G \hat{S}_{p}^{z}\left(\hat{S}_{p+\hat{x}}^{z}+\hat{S}_{p-\hat{x}}^{z}-2 \hat{S}_{p+\hat{y}}^{z}\right)\right] \\
& \stackrel{J \ll G}{\approx} V^{(2)}-2 J \sum_{p}\left(P_{p}^{\uparrow \uparrow \uparrow}+P_{p}^{\downarrow \downarrow \downarrow}\right) \hat{S}_{p}^{x},
\end{aligned}
$$

as the nonflippable configurations are separated from the flippable ones by a nonzero gap that is precisely equal to $G$. Notice that the next-to-nearest-neighbor (NNN) pair interactions $V^{(2)}$ can be also written in terms of projectors over NN spins. For $\eta=|\eta| \hat{x}, V^{(2)}$ reduces to

$$
V^{(2)}=\sum_{p} \Lambda(|\eta|)\left(P_{p}^{\uparrow \uparrow \uparrow}+P_{p}^{\downarrow \downarrow \downarrow}-P_{p}^{\uparrow \downarrow \uparrow}-P_{p}^{\downarrow \uparrow \downarrow}\right),
$$


with $\Lambda(|\eta|)=A\left[\hat{x}+a_{y}(|\eta|), \eta\right] / 2<0$. Thus, for $J \ll G$, the effective spin Hamiltonian (15) is equivalent to the RK Hamiltonian with a generalized RK potential. We name it as the "Rydberg RK" Hamiltonian on a periodic ladder.

As the original RK Hamiltonian (8), the Rydberg RK Hamiltonian (15) displays a RVBS phase that is experimentally accessible. We plot the phase diagram of Eq. (15) for varying $\lambda=\Lambda / J$, in Fig. 4(b) for the square lattice and in Fig. 4(c) for the periodic ladder, and we compare it with the one of Eq. (8). The extra potential term in Eq. (15) modifies appreciably the phase diagram only for $\lambda \gtrsim 1$; cf. Fig. 3(c). In particular, the ferromagnetic and the RVBS phases remain unchanged, as clearly evidenced by the behavior of the structure factor. The $S_{(0,0)}[z]$ dominates in the ferromagnetic phase and coincides with $S_{(\pi, \pi)}[z]$ in the RVBS phase. For $\lambda>1$, the columnar phase is substituted by a "glassy" phase, with a huge classical degeneracy imposed by $V^{(2)}$. In the 2D case, the phase transition from the ferromagnetic phase to the RVBS phase moves slightly to the right, $\lambda_{c}$ from approximately -0.6 to approximately -0.5 , as the additional potential term stabilizes the ferromagnetic phase for $\lambda<0$. The phase transition from the RVBS phase to the glassy phase still occurs around $\lambda=1$, but the model at $\lambda=1$ is no longer integrable. In Fig. 4(c), we test numerically the validity of the mapping from the Rydberg Hamiltonian Eq. (11) to the effective Rydberg RK Hamiltonian (15) for increasing values of $J$. For this test, we consider a periodic ladder of $N_{x}=6 \times 2$ atom pairs, with periodic boundary conditions along the $x$ axis, and compare via exact diagonalization the ground state of Eq. (15) with the corresponding eigenstate of Eq. (11). We fix the displacement of the atoms in the pairs to be $\eta=0.38 a_{x} \hat{x}$, and the distance of the pairs along $y$ is $a_{y} \approx 0.59 a_{x}$. With such values, we obtain $\Lambda=-0.91 C_{6} / a_{x}^{6}$ and $G=15.71 C_{6} / a_{x}^{6}$. We also consider a pinning Hamiltonian $\delta \sum_{p} S_{p}^{z}$, with $\delta=0.1 C_{6} / a_{x}^{6}$ to compare eigenstates of the two Hamiltonians consistently in the presence of degeneracies (in particular, for $J \rightarrow 0$, i.e., $\lambda \rightarrow-\infty)$. The structure factors $S_{k}[z]$ shown in Fig. 4(c) show that the RVBS can be accessed within the regime of validity $J \ll G$ of Eq. (15). In Sec. III D, we show how to form such a state via adiabatic state preparation in an experiment.

\section{Rydberg gauge theory on the square and other 2D lattices}

We now apply the same strategy explained above to engineer the 2D RK Hamiltonians defined on generic lattice geometries, e.g., on triangular, square, and hexagonal lattices. Their dual formulation, respectively, on the dual hexagonal, square, and triangular lattices can be written in a unified fashion [84] as

$$
H_{\mathrm{RK}}^{*}=-J \sum_{p}\left(P_{p}^{\uparrow \ldots \uparrow}+P_{p}^{\downarrow \cdots \downarrow}\right)\left(2 \hat{S}_{p}^{x}-\lambda\right),
$$

where the projectors $P^{\uparrow \ldots \uparrow(\downarrow \ldots \downarrow)}=\prod_{p^{\prime}=\langle p\rangle_{1}}\left[\frac{1}{2}+(-) \hat{S}_{p^{\prime}}^{z}\right]$ involve the three, four, and six NN spins to the spin at the site $p$, respectively. In order to engineer the generalized blockade conditions associated with such projectors, we exploit the NN Ising interactions arising from $V^{(1)}$, as in the example of the periodic ladder. While the dynamical implementation of the projectors for a dual hexagonal lattice follows precisely the same path as for the periodic ladder, the higher lattice coordination of square and triangular lattices introduces new conditions. In the latter case, it is not sufficient to ask that $\sum_{p^{\prime}=\langle p\rangle_{1}} A\left(p-p^{\prime}, \eta\right)=$ $0=\sum_{p^{\prime}=\langle p\rangle_{1}} B\left(p-p^{\prime}, \eta\right)$ as additional degeneracy can arise. For instance, if the Ising couplings to two neighbor spins at $p_{1}$ and $p_{2}$ are opposite, $A_{p-p_{1}}=-A_{p-p_{2}}$, we are free to flip these two spins without paying any interaction energy. Thus, the Ising interactions project at low energies also on additional unwanted configurations. In practice, in lattices with more than three neighbors, we have to design the Ising couplings $A\left(p-p^{\prime}\right), p^{\prime}=\langle p\rangle_{1}$, such that their sum for any subset $\left\{\langle p\rangle_{1}\right\}$ of NN spins is nonzero. The gap $G$ is the modulus of the smallest sum, $G=$ $\min \left[\left|\sum_{p^{\prime} \in\left\{\langle p\rangle_{1}\right\}} A\left(p-p^{\prime}, \eta\right)\right|\right]$.

For concreteness, we sketch here how to find the proper array of atoms that avoids the unwanted degeneracies and realize the Rydberg RK Hamiltonian on the square lattice. The details of the calculation are presented in Appendix D. For the construction on other lattices, we refer the reader to Ref. [84]. In what follows, we consider a rectangular lattice of pairs deformed by the relative displacement of its even and odd sublattices, indicated in blue and red in the right in Fig. 4(a). As shown in this figure, we displace the sublattices along $y$ by $d_{y} \hat{y}$ while the pairs lie in the $x z$ plane, with the relative displacement of the atoms parametrized as $\eta=|\eta|(\cos \theta \hat{x}+\sin \theta \hat{z})$. We fix the lattice spacing along $x$ to $a_{x}=1$, while the one along $y$ is $a_{y}$. For any $\theta, d_{y}$, and $a_{y}$, the constant detunings in $V^{(1)}$ add up zero. The Ising couplings to the left and right $\mathrm{NN}$ spins are the same and positive, while for $d_{y} \neq 0$ the Ising couplings to the top and to the bottom NN spins are different and negative. By adjusting $a_{y}$, we can make the sum of the four couplings zero, while any sum of two or three of them is not, and thus achieve the desired blockade condition with a finite gap $G$ to the unflippable configurations.

The effective Hamiltonian takes the RK form

$$
H_{\mathrm{Ryd}} \stackrel{J \ll G}{\approx}-2 J \sum_{p}\left(P_{p}^{\uparrow \uparrow \uparrow \uparrow}+P_{p}^{\downarrow \downarrow \downarrow \downarrow}\right) \hat{S}_{p}^{x}+V^{(2)}
$$

with the generalized RK potential determined by NNN interactions

$$
V^{(2)}=\sum_{p} \Lambda\left(P_{p}^{\uparrow \uparrow \uparrow \uparrow}+P_{p}^{\downarrow \downarrow \downarrow \downarrow}-P_{p}^{\uparrow \downarrow \uparrow \downarrow}-P_{p}^{\downarrow \uparrow \downarrow \uparrow}\right)
$$


where we order the NN spins anticlockwise and $\Lambda=\Lambda\left(\eta, d_{y}\right)$.

We can use $d_{y}$ and $\theta$ to both maximize the gap $G$ and minimize the ratio $|\Lambda / G|$. In particular, we can reduce the latter arbitrarily such that the RK Hamiltonian (18) is valid for $J \gtrsim \Lambda / \lambda_{c}$, and the driven Rydberg array supports a RVBS phase.

\section{Adiabatic preparation of the RVBS phase in decorated Rydberg arrays}

Above, we show how to engineer Rydberg arrays that naturally realize dual RK Hamiltonians on different lattice geometries. In particular, we show that the dual RK on a square lattice displays a RVBS phase. Here, we discuss how to prepare this phase and how to access the full phase diagram of such Hamiltonians adiabatically in current Rydberg experiments. The idea is to exploit the detuning of the effective Rabi coupling for the spins as done in several experiments $[4-6,8,9]$. We consider a driven array of atom pairs as in Eq. (9), where the ground states of the two atoms in the pair have an energy offset $\delta$ much smaller than both the detuning and the van der Waals interaction in the pair, $\delta \ll-\Delta, C_{6} /|\eta|^{6}$, as produced, for instance, by an optical lattice. In the spin language, the net effect of such a term is to induce a detuning for the spins such that their effective Hamiltonian in generic geometry reads

$$
\begin{aligned}
H_{\mathrm{Ryd}} & \approx \sum_{p}\left(-2 J \hat{S}_{p}^{x}+\delta \hat{S}_{p}^{z}\right)+V^{(1)}+V^{(2)} \\
& \approx \sum_{p}\left[-2 J\left(P_{p}^{\uparrow \ldots \uparrow}+P_{p}^{\downarrow \cdots \downarrow}\right) \hat{S}_{p}^{x}+\delta \hat{S}_{p}^{z}\right]+V^{(2)},
\end{aligned}
$$

where in the second line we specialize to arrays that satisfy the geometric requirements discussed in Secs. III B and III C and take the effective Rabi coupling sufficiently smaller than the gap $G, J \ll G$. The presence of the detuning does not change such geometric requirements, as it is a diagonal term that commutes with $V^{(1)}$. This result also implies that we can prepare adiabatically the ground state (or the maximally excited state) of the emergent dual RK Hamiltonian (20) for $\delta=0$ in any phase accessible within its regime of validity.

For concreteness, let us focus on the preparation of the RVBS phase on the square lattice. We consider the possibility to vary $J(t)$, for instance, via the Rabi frequency $\Omega(t)$, as a function of time. The idea is then to access the phase from the ferromagnetic phase that is connected to the product state with all the spin ups, with $J(t=0) \ll|\Lambda|$. The value of $J(t)$ is then slowly increased to reach the final state of the adiabatic state preparation. The procedure is illustrated in the case of the periodic ladder in Fig. 4(e), with a pulse $J(t)=J \sin \left[\pi t /\left(2 t_{f}\right)\right], t_{f}=40 / J$, shown in the inset. We simulate the dynamics within Eq. (11) and for the same parameters as Fig. 4(d). For the chosen value of
$J=C_{6} / a_{x}^{6}$, the system acquires a RVBS pattern at the final time $t_{f}$.

We expect the protocol to be able to form RVBS states also for larger ladders that are beyond the capability of classical computation. In realistic experimental setups, the adiabatic preparation is limited by the finite coherence time of the array due to spontaneous decay of the Rydberg states and to their motion. The inverse of the coherence time sets a minimal speed at which the parameters can change. Such a minimal speed sets the minimal energy gap (at the transition between the ferromagnetic and the RVBS phases) compatible with adiabaticity. Thus, the coherence time determines the maximal size of the ladder whose RVBS phase can be prepared adiabatically. Our current protocol, which is not optimized, requires a running time of $10 \mathrm{~J}^{-1}$, while we estimate that the coherence time can be 10 times larger. For instance, in the setup of Ref. [5] by setting $|\eta|=0.38 a_{x}=1 \mu \mathrm{m}$, we get $J=C_{6} / a_{x}^{6} \sim 20 \mathrm{MHz}$, such that the overall coherence time of the Rydberg system (estimated in Ref. [5] to be $7 \mu \mathrm{s}$ ) is of the order of $100 \mathrm{~J}^{-1}$.

A similar adiabatic protocol applies to the preparation of the RVBS phase in a $N_{x} \times N_{y}$ lattice. We fix the displacement of the atoms in the pairs to be $\eta=a_{x}(0.33 \hat{x}+0.38 \hat{z})$ and the displacement of the sublattices along $y$ to $d_{y}=$ $0.07 a_{x}$ such that the average distance of the pairs along $y$ is $a_{y}=0.88 a_{x}$. With such values, the (blue-detuned) driven array is described by the Hamiltonian (18) with $\Lambda=$ $-0.088 C_{6} / a_{x}^{6}$ and $G=1.42 C_{6} / a_{x}^{6}$. Since we get a similar ratio $\Lambda / G$ as in the periodic ladder, we expect the adiabatic preparation to work in a similar way.

\section{CONCLUSIONS AND OUTLOOK}

In this paper, we have shown that we can perform scalable quantum simulation of 2D lattice gauge theories with reconfigurable Rydberg arrays in current experiments. As a prototype of gauge theory with magnetic (plaquette) interactions, we have targeted the RK model, a spin-1/2 U (1) gauge theory that it is relevant for quantum magnetism. With the help of the electromagnetic duality, we have evidenced that the dynamics of physical states has a blockade character that it is realized by geometrically tuned Rydberg arrays. We have detailed the engineering of the dual RK Hamiltonian on the square lattice without background charges, and we have computed its phase diagram for varying Rabi couplings. We have shown how to prepare and detect the RVBS phase in Rydberg experiments with ladders and 2D arrays.

Our findings open several new possibilities for the quantum simulation of lattice gauge theories and more generally of many-body physics.

\section{A. Generalized RK Hamiltonians}

The relation we have established between the RK model and driven Rydberg arrays through the duality extends also 
to other (i) lattice geometries and (ii) charge backgrounds [84]. Furthermore, the Rydberg implementation may suggest (iii) new mechanisms to achieve $\mathrm{U}(1)$-spin liquid phases in two dimensions.

\section{Geometries}

Contrary to the quantum dimer counterpart [12], the phase diagram of the RK model without background charges has not received much attention on other lattice geometries than the square ones. The precise structure of the phase diagram is generally unknown, and we may expect surprises in the phase diagram especially for frustrated lattices that are not bipartite. The first case to examine is the RK Hamiltonian on a triangular lattice, as its dual is realized by a properly deformed hexagonal decorated Rydberg array [84].

\section{Charges}

Static background charges are especially interesting, because they allow one to probe confinement in the gauge theory and can lead to nested phases (see Fig. 6 in Ref. [70]). In the dual RK model, static background charges can be incorporated by modifying the map (5) and the blockade condition governing the plaquette flip (6). For instance, if we place a pair of two \pm 1 charges at a distance, we must include the effect of the string between the charges, which amounts to flipping the link spins along the path (for a very recent study on a special background, see Ref. [91]). Thus, the map (5) acquires a minus sign along the path of string that changes the projector in Eq. (6) along the string. Notice that, by applying the same reasoning to the staggered distribution of charges that leads to the quantum dimer model (see Appendix A), one recovers the duality to the fully frustrated Ising model first found in Ref. [86].

As a final remark, we notice that the dual approach considered here can be extended to simulate the Higgs mechanism in the untruncated $\mathrm{U}(1)$ gauge theory considering larger plaquette spins (see below) as in Ref. [92] and, perhaps, an analog version of it for fermionic matter; cf. Ref. [93]. In principle, the dual approach we consider here can be extended from static (c-number) to dynamical charges. The price to pay is that the tunneling of charges becomes nonlocal in the charges, as in the 1D Schwinger model, and in the plaquette spins. The implementation of such a term would be possible in a digital, i.e., a Trotterized, approach of the time evolution [79]. Whether such a term can be conveniently engineered within present Rydberg technology is under investigation. Alternative digital schemes that include dynamical matter can be found in Refs. [36,37].

\section{U(1)-spin liquids}

It is well known that the Coulomb phase of compact U(1) gauge theory is stable in three dimensions, while it is unstable in two dimensions due to the instanton effect [20].
This continuum argument explains a posteriori why, contrary to 3D [94,95], the RK point of the RK model in $2 \mathrm{D}$ does not extend to a spin liquid phase on bipartite lattices, which is instead replaced by a RVBS phase that is confined. The absence of a deconfined "photon" excitation that rules out the existence of a spin liquid phase can be circumvented by breaking translational invariance as happens with Cantor deconfinement [96-98]. It is very interesting to explore whether in a similar spirit we can break translational invariance such to induce and stabilize a spin liquid phase in 2D, e.g., by considering additional interaction terms to the dual RK model that are natural from the Rydberg perspective. Alternatively, it is intriguing to explore the realization of a 3D spin liquid phase or of magnetic monopoles [99] in 3D Rydberg arrays.

\section{B. New experimental probes}

Our approach to quantum simulations of the dual RK model allows one (i) to access experimentally quantum correlations and (ii) to probe excitations' spectrum and thermalization of the RK model through quantum quenches, beyond condensed matter experiments and classical computations.

\section{Quantum correlations}

Contrary to traditional measurement schemes in condensed matter, Rydberg simulators allow for single-site resolution to detect whether each atom is in the ground or excited state. On the one hand, such a possibility allows us to characterize the phase diagram for different values of $\lambda$ through the structure factor and the expectation value of the generalized RK potential, which are experimentally accessible observables. On the other hand, it allows us to access nonlocal order parameters like the Wilson loop or to measure directly the entanglement entropy by quantum interference as in Ref. [100] or by random measurements as in Ref. [101] (for the theory proposal, see Refs. [102-104] and Ref. [105], respectively). An alternative promising route to characterize quantum many-body states is to use quantum machine learning techniques, e.g., for performing quantum many-body state reconstruction efficiently as proposed in Ref. [106] and experimentally realized in Ref. [107], or to identify phases with an unknown, nonlocal order parameter [108].

\section{Dynamical probes}

Static quantum correlations discussed above can be potentially determined numerically, for instance, by quantum Monte Carlo or by DMRG for sufficiently large lattices such to approach the thermodynamic limit; see Refs. [70,109] for recent calculations. A key advantage of the quantum simulators is that is equally easy to study the time evolution of the observables after a local or a global quench [110]. These experiments could shed light 
on both the excitations above the ground state and the thermalization properties in the gauge theory. In particular, it would be very interesting to study whether the relation between confinement and many-body quantum scars [5,111,112], first noted for Rydberg chains and the 1D Schwinger model [19], extends to 2D pure gauge theories (for the dynamical phase transition in the Schwinger model, see Ref. [113]). Recent numerical studies with exact diagonalization in 2D spin-1/2 U(1) gauge theory without charges in Ref. [114] and in the quantum dimer model and Ref. [115] have found evidences on small lattices of dynamical phase transitions (the emergence of kinklike structures in the return amplitude to the original ground state manifold) for quantum quenches from flippable to RVBS phase and the emergence of glassy behavior (very slow relaxation time) for quantum quenches from flippable to the unflippable phase, respectively. The latter behavior have been very recently confirmed and related to the constraint dynamics of the dimers in Ref. [116].

\section{Exotic spin models}

The use of configurable Rydberg arrays of atom pairs induces effective interacting spin-1/2 Hamiltonians with tunable couplings between spins. This is precisely the technique we have employed in the quantum simulation of the RK model. Following a similar strategy and clustering together $2 S+1$ atoms in a macro-atom, we can form composite spins $S$ whose spin-spin interactions are still controlled by the arrangements of the macro-atoms and their relative displacements. Therefore, configurable Rydberg arrays can be used for programable simulations of exotic spin- $S$ models with anisotropic interactions. We expect the local competition between ferromagnetic and antiferromagnetic interactions to lead to novel entangled phases and behavior. Additionally, the generalized blockade conditions that emerge in the simulator can encode known as well as novel "quantum" cellular automata [117]. The complexity generated by arranging driven Rydberg arrays in clusters can be viewed as the dynamical counterpart of quantum optimization for the maximum independent set studied in Ref. [121]. We foresee that the combination of clustering of the arrays with angulardependent Rydberg-Rydberg interactions and/or with more complex Rydberg series such as those associated with earth-alkali atoms [122-124] will open even more exciting perspectives for quantum simulation.

\section{ACKNOWLEDGMENTS}

A. C. thanks D. Gonzalez Cuadra for deriving the dual form of the plaquette operator of the spin- $1 / 2$ gauge theory on an open ladder and P. Silvi for discussions on the formulation of the same duality on square lattice. We thank A. Keesling and W. W. Ho for useful discussions and M. Dalmonte for insightful comments on an advanced version of the manuscript. Research in Innsbruck is supported by the European Research Council (ERC) under the European Union's Horizon 2020 research and innovation programme (Grant Agreement No. 741541), and from the European Union's Horizon 2020 research and innovation programme under Grant Agreements No. 817482 (Pasquans) and No. 731473 (FWF QuantERA via QTFLAG I03769). Furthermore, this work was supported by the Simons Collaboration on UltraQuantum Matter, which is a grant from the Simons Foundation (651440, P. Z.). Research in Harvard is supported by the Center for Ultracold Atoms, the National Science Foundation, the U.S. Department of Energy, and the Office of Naval Research. A.C. acknowledges support from the Universitat Autònoma de Barcelona Talent Research program, from the Ministerio de Economía y Competividad MINECO (Contract No. FIS2017-86530-P), and from the the European Regional Development Fund (ERDF) within the ERDF Operational Program of Catalunya (project QUASICAT/QuantumCat). O. V. acknowledges support from Real Colegio Complutense Harvard and Fundacion Ramon Areces.

\section{APPENDIX A: SPIN GAUGE THEORY AS DIMER MODEL}

In this section, we detail the relation between the spin$1 / 2 \mathrm{U}(1)$ gauge theory and the quantum dimer model on a square lattice. First of all, we notice that by applying a $\hat{S}^{x}$ transformation on the links at the bottom-left corner, $(s, x)$ and $(s, y)$, or on the links at the top-right one, $(s+\hat{x}, y)$ and $(s+\hat{y}, x)$, the plaquette operator on the plaquette $s$ assumes a ring exchange form with alternated spin flips:

$$
\hat{\mathcal{S}}_{s}^{\dagger}+\hat{\mathcal{S}}_{s}=\hat{S}_{s, x}^{+} \hat{S}_{s+\hat{x}, y}^{-} \hat{S}_{s+\hat{y}, x}^{+} \hat{S}_{s, y}^{-}+\text {H.c. }
$$

As a consequence, the flippable plaquette configurations become the ones with alternated spins, i.e., the ones with parallel colored links; see Fig. 5(a). By extending the transformation to the full 2D plane, for instance, by reverting the bottom-left corner of the odd plaquettes (= the top-right corner of the even ones), as shown in Fig. 5(b), the RK Hamiltonian is still of the form (4), with the plaquette operator given in Eq. (A1), while the Gauss law (2) takes becomes

$$
\hat{E}_{s, x}+\hat{E}_{s, y}+\hat{E}_{s-\hat{x}, x}+\hat{E}_{s-\hat{y}, y}+(-1)^{s} \hat{Q}_{s}=0,
$$

where $(-1)^{s} \equiv-1^{m+n}$, where $m, n$ are the Cartesian coordinates of the site $s=m \hat{x}+n \hat{y}$. In Fig. 5(b), we show that the background $|\Omega\rangle$ becomes a columnar state, the fully flippable background in the new basis.

The quantum dimer model is obtained by considering the physical states with staggered background charges. For instance, by taking $Q_{s}=(-1)^{s}$, to each site is attached only one dimer that is identified by the colored link in $|\uparrow\rangle$, 
(a)

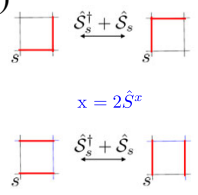

(b)

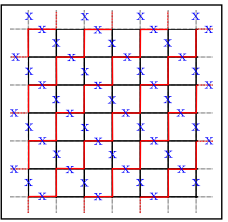

(c)

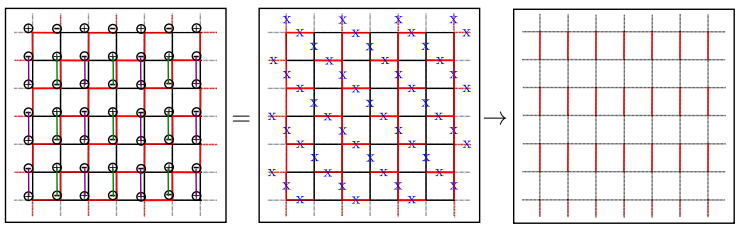

FIG. 5. Relation between gauge magnets and the quantum dimer model. (a) By reversing the spins of the links at the bottomleft (or at the top-right) corner of the plaquette, the plaquette operator becomes a dimer move; see Eq. (A1). (b) In the dimer basis, obtained by reversing the spins of the links at the bottomleft corner of the odd plaquettes, the fully flippable background $|\Omega\rangle$ appears as a columnar state. (c) The physical states of the quantum dimer model are obtained for a staggered distribution of static charges, $Q_{s}=(-1)^{s}$. Such states can be constructed by applying the plaquette operator on the maximally flippable background depicted in the figure. It is obtained by superimposing a string covering associated to the charges on $|\Omega\rangle$. Because of the charges, only half of the plaquettes are flippable.

while the empty ones are in $|\downarrow\rangle$. In Fig. 5(c), we represent one of the maximally flippable configurations of the quantum dimer model: Only half of the plaquettes are flippable. It can be visualized as the result of a special string covering superimposed over $|\Omega\rangle$. The string connecting the charges block at least half of the plaquette and change the physical states. Several properties distinguish the physical states of the quantum dimer models from the physical states without background charges. Although they share the same Hamiltonian (A1), the different boundary conditions determine a different phase diagram and a different dual Hamiltonian for the two models.

\section{APPENDIX B: ELECTRIC TRUNCATION AS A DYNAMICAL PROCESS AND RELATION WITH KOGUT-SUSSKIND U(1)}

We analyze here the dynamics of spin gauge theories in comparison to the nontruncated U(1) gauge theory. For simplicity, we explicitly discuss the pure gauge case, but the analysis applies also in the presence of dynamical matter. We start by rewriting the pure gauge Kogut-Susskind Hamiltonian (1) in the rotating frame of the electric term:

$H_{\mathrm{KS}}^{\prime}(t)=\frac{-1}{2 g^{2}} \sum_{s} \hat{V}_{s, x}^{\dagger}(t) \hat{V}_{s+\hat{x}, y}^{\dagger}(t) \hat{V}_{s+\hat{y}, x}(t) \hat{V}_{s, y}(t)+$ H.c.,

where $\quad V_{s, \mu}^{(\dagger)}(t)=\exp \left[\left(-i g^{2} / 2\right) \hat{E}_{s, \mu}^{2}\right] \hat{U}_{s, \mu}^{(\dagger)} \exp \left[\left(i g^{2} / 2\right) \hat{E}_{s, \mu}^{2}\right]$, $\mu=x, y$. Since $\hat{U}_{s, \mu}^{(\dagger)}$ decreases (increases) the electric field

by one, the magnetic couplings between highly excited electric states are fast rotating. Thus, in the rotating frame the usual competition between the electric and magnetic translates in a soft constraint for the plaquette operator that dynamically suppresses the coupling between highly excited electric states.

We can obtain the truncated theory dynamically from Eq. (B1) by deforming the electric term. For instance, we can achieve the spin $-\frac{1}{2}$ link model Hamiltonian by first shifting the electric eigenvalues by $\frac{1}{2}$ [which is equivalent to including a $\theta$ term equal to $\pi$ in the $\mathrm{U}(1)$ Hamiltonian] and then considering a rescaled electric term $\left(g^{2} / 2\right) \kappa \times$ $\sum_{s}\left(\hat{E}_{s, x}^{2}+\hat{E}_{s, y}^{2}-\frac{1}{2}\right)$. In the limit of $\kappa \rightarrow \infty$, the electric states with $|E|>\frac{1}{2}$ decouple, and the plaquette operator reduces to Eq. (3). Such a modification is equivalent to replacing the quadratic electric term with one of a box potential form. Although the electric term acts as the identity on the remaining electric state, its effect on the dynamics persists: The plaquette operators on neighboring plaquettes do not compute due to the truncation of the electric states. The electric truncation in spin gauge theories is equivalent to a hard version of the energy penalty due to the electric interactions. The competition between electric and magnetic interactions that characterizes gauge theories in more than one dimension survives in the truncated theories. The spin- $\frac{1}{2}$ link models we study here are, thus, the minimal instance of such gauge theories with continuous gauge groups.

\section{APPENDIX C: CONSTRUCTION OF THE DUALITY}

Without charges, all gauge-invariant electric states of the U(1) Kogut-Susskind lattice gauge theory can be written as combinations of closed electric loops, i.e., closed oriented paths of links with a constant electric field (with respect to the path orientation). With the exclusion of "large" topologically nontrivial loops, every other loop configuration and, thus, all physical states can be written as a combination of elementary plaquette loops. The electric field of a loop on the plaquette $p$ (conventionally oriented anticlockwise) assumes the meaning of a potential, and it is called height, $h_{p}$. In two dimensions, the electric field on each link is given by the difference of the heights of the two plaquettes sharing that link (according to the standard lattice orientation, it is the difference between the left and right heights). Therefore, the electric field operator can be written as an operator relation

$$
\begin{aligned}
& \hat{E}_{p, x} \rightarrow \hat{h}_{p}-\hat{h}_{p-\hat{y}}, \\
& \hat{E}_{p, y} \rightarrow \hat{h}_{p-\hat{x}}-\hat{h}_{p},
\end{aligned}
$$

which defines the dual theory on the plaquette basis. The heights can take any integer value, and there is a unique identification between the heights and the electric states on the links, up to the definition of the overall height origin. 
The Kogut-Susskind Hamiltonian (1) in the dual basis simplifies considerably as the plaquette operator $\hat{\mathcal{U}}_{p}+$ $\hat{\mathcal{U}}_{p}^{\dagger}=\hat{U}_{p, x} \hat{U}_{p+\hat{x}, y} \hat{U}_{p+\hat{y}, x}^{\dagger} \hat{U}_{p, y}^{\dagger}+$ H.c. acts on the plaquette $p$ by raising and lowering the height $h_{p}$ by one:

$$
\left[\hat{h}_{p}, \hat{\mathcal{U}}_{p}^{(\dagger)}\right]=-(+) \hat{\mathcal{U}}_{p}^{(\dagger)} .
$$

The height construction holds also in a truncated theory like the spin-1/2 gauge theory described in Sec. II A, with the only difference that the truncation limits also the admissible height values. Since the value of the electric field on the links and, thus, the difference between the neighboring heights is limited to $\pm \frac{1}{2}$, the heights are identified mod 2 and can be represented as components of a spin 1/2. Thus, we can replace Eq. (C1) with Eq. (5) given in Sec. II B.

Any allowed raising or lowering of the height is identified with a flip of the dual spin; thus, from Eq. (C2), we have $\hat{\mathcal{U}}_{p}, \hat{\mathcal{U}}_{p}^{\dagger} \rightarrow 2 \hat{S}_{p}^{x}$. Furthermore, we can write the plaquette operator in the truncated theory as the KogutSusskind one projected on the allowed electric states:

$$
\begin{aligned}
\mathcal{S}_{p}+\mathcal{S}_{p}^{\dagger}= & 2 S_{p}^{x}\left(\frac{1}{2}-\hat{S}_{p, x}^{z}\right)\left(\frac{1}{2}-\hat{S}_{p+\hat{x}, y}^{z}\right) \\
& \times\left(\frac{1}{2}+\hat{S}_{p+\hat{y}, x}^{z}\right)\left(\frac{1}{2}+\hat{S}_{p, y}^{z}\right)+\text { H.c. }
\end{aligned}
$$

By expressing the projectors on the links in terms of the plaquette spins through Eq. (5), we find Eq. (6).

\section{APPENDIX D: DETAILS ON THE RYDBERG IMPLEMENTATION}

Here, we detail the implementation of the blockade condition on the square lattice. For the array geometry illustrated in the left in Fig. 4(a), we have by construction that $\sum_{p^{\prime}=\langle p\rangle_{1}} B\left(p-p^{\prime}, \eta\right)=0$. Indeed, from Eq. (14), it follows that $B\left[\left(a_{y} \pm d_{y}\right) \hat{y}, \eta\right]=0$, because the displacement is in the $x z$ plane and $\eta \cdot \hat{y}=0$, and $B\left(-\hat{x} \pm d_{y} \hat{y}, \eta\right)=-B\left(\hat{x} \pm d_{y} \hat{y}, \eta\right)$. The Ising couplings to the left and right NN spins are the same, $A\left(-\hat{x} \pm d_{y} \hat{y}, \eta\right)=A\left(+\hat{x} \pm d_{y} \hat{y}, \eta\right)$, while the ones to the top and to the bottom NN spins are different, $A\left[\left(-a_{y} \pm d_{y}\right) \hat{y}, \eta\right] \neq A\left[\left(-a_{y} \pm d_{y}\right) \hat{y}, \eta\right]$, for $d_{y} \neq 0$. Thus, we can achieve the desired generalized blockade condition by solving

$-2 A\left(+\hat{x}+d_{y} \hat{y}, \eta\right)=A\left[\left(a_{y}-d_{y}\right) \hat{y}, \eta\right]+A\left[\left(a_{y}+d_{y}\right) \hat{y}, \eta\right]$,

for $a_{y}=a_{y}\left(\eta, d_{y}\right)$. The solution exists for a wide range of the parameters $|\eta|, \theta$, and $d_{y}$. The function $a_{y}\left(\eta, d_{y}\right)$ cannot be written in closed form and has to be calculated numerically. Through $a_{y}\left(\eta, d_{y}\right)$, we can calculate both the gap

$$
\begin{aligned}
& G\left(\eta, d_{y}\right)=\min \left[A\left\{\left[a_{y}\left(\eta, d_{y}\right)+\left|d_{y}\right|\right] \hat{y}, \eta\right\},\right. \\
& \left.\frac{1}{2}\left(A\left\{\left[a_{y}\left(\eta, d_{y}\right)-\left|d_{y}\right|\right] \hat{y}, \eta\right\}-A\left\{\left[a_{y}\left(\eta, d_{y}\right)+\left|d_{y}\right|\right] \hat{y}, \eta\right\}\right)\right],
\end{aligned}
$$

which determines the regime of validity of the effective RK Hamiltonian (18) and the coefficient of the modified RK potential (19):

$$
\Lambda\left(\eta, d_{y}\right)=A\left[x+a_{y}\left(\eta, d_{y}\right), \eta\right] .
$$

The best-suited values of the free parameters $|\eta|, \theta$, and $d_{y}$ are obtained through the requirements that (i) the spin effective description of the atom pair is valid, (ii) the gap is maximal, such that the overall energy scale of the effective Hamiltonian is as high as possible compared to the inverse decoherence time, and (iii) the ratio $\Lambda / G$ is sufficiently small such that we can access the RVBS phase within the validity of the effective Hamiltonian (18). Since the intrapair and outer-pair separation of the atoms strongly depends on the modulus of the displacement $|\eta|$, the first requirement sets an upper bound on $|\eta|$. This bound depends weakly on $\theta$ and $d_{y}$, and it is an increasing function of the former and decreasing function of the latter. Since the gap $G$ strongly depends on $d_{y}$, the second requirement fixes an optimal value for $d_{y}$ that is weakly increasing function $|\eta|$ and $\theta$. The value of $\Lambda$ strongly depends on $\theta$ : In fact, as it happens for dipolar interactions there exists a magic angle for which it is exactly zero. However, also the gap $G$ depends $\theta$ in a similar way: In this case, the magic angle is smaller. In other words, we can diminish the ratio $\Lambda / G$ and satisfy the third requirement by choosing sufficiently large $\theta$. An optimal compromise between a large gap $G$ and small $\Lambda / G$ ratio is obtained for $|\eta|=0.5 a_{x}, d_{y}=0.07 a_{x}$, and $\theta=0.85 \mathrm{rad}$, which gives $a_{y}=0.88 a_{x}, G=1.42 C_{6} / a_{x}^{6}$, and $\Lambda=-0.088 C_{6} / a_{x}^{6}$. Thus, at the price of reducing the overall energy scale (by an order of magnitude in relative terms), we can achieve a similar ratio $\Lambda / G<(1 / 16)$ as in the periodic ladder and ensure the existence of an accessible RVBS phase. In absolute terms, by considering the setup of Ref. [5] and by setting $|\eta|=0.5 a_{x}=1 \mu \mathrm{m}$ and $J=0.16 C_{6} / a_{x}^{6} \sim$ $16 \mathrm{MHz}$, we estimate a coherence time (in Ref. [5] of $7 \mu \mathrm{s}$ ) of the order of $80 \mathrm{~J}^{-1}$.

\section{APPENDIX E: DETAILS ON THE DMRG SIMULATIONS}

Here, we provide details on the DMRG simulations that were realized using the ITensor library. The 2D indices $\left(i_{x}\right.$, $i_{y}$ ) entering the constructing of matrix-product operators are ordered as $l=i_{y}+\left(i_{x}-1\right) N_{y}$ for $\bmod \left(i_{x}, 2\right)=1$ and 
$l=N_{y}+1-i_{y}+\left(i_{x}-1\right) N_{y}$ for $\bmod \left(i_{x}, 2\right)=0$. We consider periodic boundary conditions along the $y$ axis.

We used a small pinning field $\tilde{\delta}=0.1 J$ along the $z$ direction, on three sites $\left(i_{x}, i_{y}\right)=(1,1),(2,1),(2,2)$ to favor one ground state in the case of degeneracies (in particular, for $\lambda \rightarrow-\infty$ ). We also impose an energy penalty term to select only ground states within the physical subspace

$$
\begin{aligned}
H_{\text {pen }}= & E \sum_{p}\left(P_{p}^{\downarrow} P_{p+x}^{\downarrow} P_{p+y}^{\uparrow} P_{p+x+y}^{\uparrow}+P_{p}^{\downarrow} P_{p+x}^{\uparrow} P_{p+y}^{\downarrow} P_{p+x+y}^{\uparrow}\right. \\
& \left.+P_{p}^{\uparrow} P_{p+x}^{\uparrow} P_{p+y}^{\downarrow} P_{p+x+y}^{\downarrow}+P_{p}^{\uparrow} P_{p+x}^{\downarrow} P_{p+y}^{\uparrow} P_{p+x+y}^{\downarrow}\right),
\end{aligned}
$$

with $P_{p}^{\uparrow, \downarrow}=\left(1 / 2 \pm S_{p}^{z}\right)$. One can check that the states that can be reached by the plaquette operators from the vacuum $|\Omega\rangle$, i.e., that satisfy the Gauss law in the dual formulation, satisfy $\left\langle H_{\text {pen }}\right\rangle=0$. In our simulations, we use $E=5 \mathrm{~J}$ and $E=10 J$.

Finally, we achieve ground state convergence, below the percent level with respect to spin-spin correlations, for maximum bond dimensions $D=256$.

\section{APPENDIX F: GROUND STATE OF THE PXP MODEL}

In this section, we show results for the ground state of the 1D PXP model

$$
H_{1 \mathrm{D}}=\sum_{p} P_{p-\hat{x}}^{\uparrow} P_{p+\hat{x}}^{\uparrow}\left(-2 S_{p}^{x}+\lambda\right)
$$

calculated via the DMRG, with open boundary conditions, and with a small pinning field $\tilde{\delta}=0.1 J$ on the first site. We also impose the blockade constraint $\sum_{p}\left\langle P_{p}^{\downarrow} P_{p+x}^{\downarrow}\right\rangle$ by energy penalty.

The phase diagram in terms of structure factors is represented in Fig. 6 and shows no signatures of a RVBS phase.
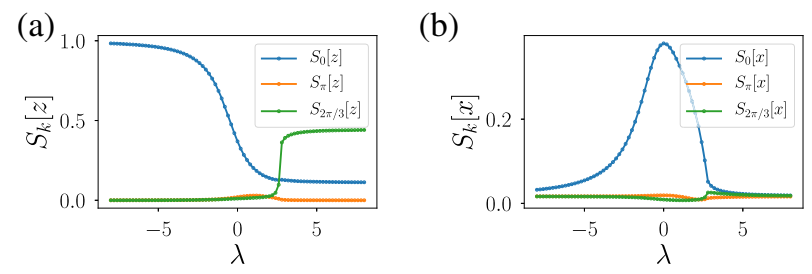

FIG. 6. The ground state of the 1D PXP model. (a) Structure factors for $z$ spin-spin correlations for $k=0, \pi, 2 \pi / 3$. (b) Structure factors for $x$ spin-spin correlations for $k=0, \pi, 2 \pi / 3$. Parameters: $N_{x}=60$ and maximum bond dimension $D=128$.
[1] M. Endres, H. Bernien, A. Keesling, H. Levine, E. R. Anschuetz, A. Krajenbrink, C. Senko, V. Vuletic, M. Greiner, and M.D. Lukin, Atom-by-Atom Assembly of Defect-Free One-Dimensional Cold Atom Arrays, Science 354, 1024 (2016).

[2] D. Barredo, S. de Léséleuc, V. Lienhard, T. Lahaye, and A. Browaeys, An Atom-by-Atom Assembler of Defect-Free Arbitrary Two-Dimensional Atomic Arrays, Science 354, 1021 (2016).

[3] D. Barredo, V. Lienhard, S. de Léséleuc, T. Lahaye, and A. Browaeys, Synthetic Three-Dimensional Atomic Structures Assembled Atom by Atom, Nature (London) 561, 79 (2018).

[4] H. Labuhn, D. Barredo, S. Ravets, S. de Léséleuc, T. Macrì, T. Lahaye, and A. Browaeys, Tunable Two-Dimensional Arrays of Single Rydberg Atoms for Realizing Quantum Ising Models, Nature (London) 534, 667 (2016).

[5] H. Bernien et al., Probing Many-Body Dynamics on a 51 Atom Quantum Simulator, Nature (London) 551, 579 (2017).

[6] V. Lienhard, S. de Léséleuc, D. Barredo, T. Lahaye, A. Browaeys, M. Schuler, L.-P. Henry, and A. M. Läuchli, Observing the Space- and Time-Dependent Growth of Correlations in Dynamically Tuned Synthetic Ising Models with Antiferromagnetic Interactions, Phys. Rev. X 8, 021070 (2018).

[7] S. de Lésĺeuc, V. Lienhard, P. Scholl, D. Barredo, S. Weber, N. Lang, H. P. Büchler, T. Lahaye, and A. Browaeys, Experimental Realization of a Symmetry Protected Topological Phase of Interacting Bosons with Rydberg Atoms, Science 365, 775 (2019).

[8] P. Schauss, J. Zeiher, T. Fukuhara, S. Hild, M. Cheneau, T. Macri, T. Pohl, I. Bloch, and C. Gross, Crystallization in Ising Quantum Magnets, Science 347, 1455 (2015).

[9] E. Guardado-Sanchez, P. T. Brown, D. Mitra, T. Devakul, D. A. Huse, P. Schauß, and W.S. Bakr, Probing the Quench Dynamics of Antiferromagnetic Correlations in a 2D Quantum Ising Spin System, Phys. Rev. X 8, 021069 (2018).

[10] A. Browaeys, D. Barredo, and T. Lahaye, Experimental Investigations of Dipole-Dipole Interactions between a Few Rydberg Atoms, J. Phys. B 49, 152001 (2016).

[11] D. S. Rokhsar and S. A. Kivelson, Superconductivity and the Quantum Hard-Core Dimer Gas, Phys. Rev. Lett. 61, 2376 (1988).

[12] R. Moessner and K. S. Raman, Quantum Dimer Models, in Introduction to Frustrated Magnetism, Springer Series in Solid-State Sciences Vol. 164, edited by C. Lacroix, P. Mendels, and F. Mila (Springer, Berlin, 2011).

[13] L. Pauling, A Resonating-Valence-Bond Theory of Metals and Intermetallic Compounds, Proc. R. Soc. A 196, 343 (1949).

[14] P. W. Anderson, Resonating Valence Bonds: A New Kind of Insulator?, Mater. Res. Bull. 8, 153 (1973).

[15] P. Fazekas and P. W. Anderson, On the Ground State Properties of the Anisotropic Triangular Antiferromagnet, Philos. Mag. 30, 423 (1974).

[16] E. A. Martinez et al., Real-Time Dynamics of Lattice Gauge Theories with a Few-Qubit Quantum Computer, Nature (London) 534, 516 (2016). 
[17] C. Kokail et al., Self-Verifying Variational Quantum Simulation of Lattice Models, Nature (London) 569, 355 (2019).

[18] N. Klco, E. F. Dumitrescu, A. J. McCaskey, T. D. Morris, R. C. Pooser, M. Sanz, E. Solano, P. Lougovski, and M. J. Savage, Quantum-Classical Computation of Schwinger Model Dynamics Using Quantum Computers, Phys. Rev. A 98, 032331 (2018).

[19] F. M. Surace, P. P. Mazza, G. Giudici, A. Lerose, A. Gambassi, and M. Dalmonte, Lattice Gauge Theories and String Dynamics in Rydberg Atom Quantum Simulators, arXiv:1902.09551 [Phys. Rev. X (to be published)].

[20] A. M. Polyakov, Gauge Fields and Strings, Contemporary Concepts in Physics, Vol. 3 (Harwood Academic Publishers, Chur, 1987), p. 1, ISBN-13: 978-3718603930.

[21] X. G. Wen, Topological Orders in Rigid States, Int. J. Mod. Phys. B 04, 239 (1990).

[22] D. Horn, Finite Matrix Models with Continuous Local Gauge Invariance, Phys. Lett. B 100, 149 (1981).

[23] P. Orland and D. Rohrlich, Lattice Gauge Magnets: Local Isospin from Spin, Nucl. Phys. B338, 647 (1990).

[24] S. Chandrasekharan and U.-J. Wiese, Quantum Link Models: A Discrete Approach to Gauge Theories, Nucl. Phys. B492, 455 (1997).

[25] R. Moessner, S. L. Sondhi, and E. Fradkin, Short-Ranged Resonating Valence Bond Physics, Quantum Dimer Models, and Ising Gauge Theories, Phys. Rev. B 65, 024504 (2001).

[26] J. Kogut and L. Susskind, Hamiltonian Formulation of Wilson's Lattice Gauge Theories, Phys. Rev. D 11, 395 (1975).

[27] E. Zohar, J. I. Cirac, and B. Reznik, Simulating Compact Quantum Electrodynamics with Ultracold Atoms: Probing Confinement and Nonperturbative Effects, Phys. Rev. Lett. 109, 125302 (2012).

[28] D. Banerjee, M. Dalmonte, M. Müller, E. Rico, P. Stebler, U.-J. Wiese, and P. Zoller, Atomic Quantum Simulation of Dynamical Gauge Fields Coupled to Fermionic Matter: From String Breaking to Evolution after a Quench, Phys. Rev. Lett. 109, 175302 (2012).

[29] A. W. Glaetzle, M. Dalmonte, R. Nath, I. Rousochatzakis, R. Moessner, and P. Zoller, Quantum Spin-Ice and Dimer Models with Rydberg Atoms, Phys. Rev. X 4, 041037 (2014).

[30] V. Kasper, F. Hebenstreit, F. Jendrzejewski, M. K. Oberthaler, and J. Berges, Implementing Quantum Electrodynamics with Ultracold Atomic Systems, New J. Phys. 19, 023030 (2017).

[31] O. Dutta, L. Tagliacozzo, M. Lewenstein, and J. Zakrzewski, Toolbox for Abelian Lattice Gauge Theories with Synthetic Matter, Phys. Rev. A 95, 053608 (2017).

[32] L. Cardarelli, S. Greschner, and L. Santos, Hidden Order and Symmetry Protected Topological States in Quantum Link Ladders, Phys. Rev. Lett. 119, 180402 (2017).

[33] T. V. Zache et al., Quantum Simulation of Lattice Gauge Theories Using Wilson Fermions, Quantum Sci. Technol. 3, 034010 (2018).

[34] L. Tagliacozzo, A. Celi, A. Zamora, and M. Lewenstein, Optical Abelian Lattice Gauge Theories, Ann. Phys. (Amsterdam) 330, 160 (2013).
[35] L. Tagliacozzo, A. Celi, P. Orland, M. W. Mitchell, and M. Lewenstein, Simulation of Non-Abelian Gauge Theories with Optical Lattices, Nat. Commun. 4, 2615 (2013).

[36] E. Zohar, A. Farace, B. Reznik, and J. I. Cirac, Digital Lattice Gauge Theories, Phys. Rev. A 95, 023604 (2017).

[37] J. Bender, E. Zohar, A. Farace, and J. I. Cirac, Digital Quantum Simulation of Lattice Gauge Theories in Three Spatial Dimensions, New J. Phys. 20, 093001 (2018).

[38] U.-J. Wiese, Ultracold Quantum Gases and Lattice Systems: Quantum Simulation of Lattice Gauge Theories, Ann. Phys. (Amsterdam) 525, 777 (2013).

[39] E. Zohar, J. I. Cirac, and B. Reznik, Quantum Simulations of Lattice Gauge Theories Using Ultracold Atoms in Optical Lattices, Rep. Prog. Phys. 79, 014401 (2016).

[40] M. Dalmonte and S. Montangero, Lattice Gauge Theory Simulations in the Quantum Information Era, Contemp. Phys. 57, 388 (2016).

[41] L. Barbiero, C. Schweizer, M. Aidelsburger, E. Demler, N. Goldman, and F. Grusdt, Coupling Ultracold Matter to Dynamical Gauge Fields in Optical Lattices: From FluxAttachment to Z2 Lattice Gauge Theories, Sci. Adv. 5, eaav7444 (2019).

[42] S. Greschner, G. Sun, D. Poletti, and L. Santos, DensityDependent Synthetic Gauge Fields Using Periodically Modulated Interactions, Phys. Rev. Lett. 113, 215303 (2014).

[43] L. W. Clark, B. M. Anderson, L. Feng, A. Gaj, K. Levin, and C. Chin, Observation of Density-Dependent Gauge Fields in a Bose-Einstein Condensate Based on Micromotion Control in a Shaken Two-Dimensional Lattice, Phys. Rev. Lett. 121, 030402 (2018).

[44] F. Görg, K. Sandholzer, J. Minguzzi, R. Desbuquois, M. Messer, and T. Esslinger, Realisation of DensityDependent Peierls Phases to Couple Dynamical Gauge Fields to Matter, Nat. Phys. 15, 1161 (2019).

[45] C. Schweizer, F. Grusdt, M. Berngruber, L. Barbiero, E. Demler, N. Goldman, I. Bloch, and M. Aidelsburger, Floquet Approach to Z2 Lattice Gauge Theories with Ultracold Atoms in Optical Lattices, Nat. Phys. 15, 1168 (2019).

[46] H.-N. Dai, B. Yang, A. Reingruber, H. Sun, X.-F. Xu, Yu.-A. Chen, Z.-S. Yuan, and J.-W. Pan, Four-Body RingExchange Interactions and Anyonic Statistics within a Minimal Toric-Code Hamiltonian, Nat. Phys. 13, 1195 (2017).

[47] Recent experiments [48] show that one million atom arrays may become possible in the foreseeable future.

[48] D. O. de Mello, D. Schäffner, J. Werkmann, T. Preuschoff, L. Kohfahl, M. Schlosser, and G. Birkl, Defect-Free Assembly of 2D Clusters of More Than 100 Single-Atom Quantum Systems, Phys. Rev. Lett. 122, 203601 (2019).

[49] T. Graß, M. Lewenstein, and A. Bermudez, Dual TrappedIon Quantum Simulators: An Alternative Route towards Exotic Quantum Magnets, New J. Phys. 18, 033011 (2016).

[50] F. J. Wegner, Duality in Generalized Ising Models and Phase Transitions without Local Order Parameters, J. Math. Phys. (N.Y.) 12, 2259 (1971). 
[51] E. Fradkin and L. Susskind, Order and Disorder in Gauge Systems and Magnets, Phys. Rev. D 17, 2637 (1978).

[52] E. Fradkin and S. H. Shenker, Phase Diagrams of Lattice Gauge Theories with Higgs Fields, Phys. Rev. D 19, 3682 (1979).

[53] K. Kasamatsu, I. Ichinose, and T. Matsui, Atomic Quantum Simulation of the Lattice Gauge-Higgs Model: Higgs Couplings and Emergence of Exact Local Gauge Symmetry, Phys. Rev. Lett. 111, 115303 (2013).

[54] A. Bazavov, Y. Meurice, S.-W. Tsai, J. Unmuth-Yockey, and J. Zhang, Gauge-Invariant Implementation of the Abelian-Higgs Model on Optical Lattices, Phys. Rev. D 92, 076003 (2015).

[55] D. Gonzalez-Cuadra, E. Zohar, and J. I. Cirac, Quantum Simulation of the Abelian-Higgs Lattice Gauge Theory with Ultracold Atoms, New J. Phys. 19, 063038 (2017).

[56] K. G. Wilson, Confinement of Quarks, Phys. Rev. D 10, 2445 (1974).

[57] R. Brower, S. Chandrasekharan, and U.-J. Wiese, $Q C D$ as a Quantum Link Model, Phys. Rev. D 60, 094502 (1999).

[58] L. Tagliacozzo, A. Celi, and M. Lewenstein, Tensor Networks for Lattice Gauge Theories with Continuous Groups, Phys. Rev. X 4, 041024 (2014).

[59] The electric state degeneracy caused by a shift of the electric field of $\frac{1}{2}$ is equivalent to the presence of a $\theta$ term equal to $\pi$ in QED. Such a shift favors ground state degeneracy and thus frustration; see Ref. [60].

[60] E. Fradkin, Field Theories of Condensed Matter Physics (Cambridge University Press, Cambridge, England, 2013).

[61] P. W. Anderson, The Resonating Valence Bond State in $\mathrm{La}_{2} \mathrm{CuO}_{4}$ and Superconductivity, Science 235, 1196 (1987).

[62] D. Chowdhury and S. Sachdev, The Enigma of the Pseudogap Phase of the Cuprate Superconductors, Quantum Criticality in Condensed Matter (World Scientific, 2015), pp. 1-43, https://doi.org/10.1142/ 9789814704090_0001.

[63] S. Sachdev, Emergent Gauge Fields and the High Temperature Superconductors, Phil. Trans. R. Soc. A 374, 20150248 (2016).

[64] G. Baskaran and P. W. Anderson, Gauge Theory of HighTemperature Superconductors and Strongly Correlated Fermi Systems, Phys. Rev. B 37, 580 (1988).

[65] E. Fradkin and S. A. Kivelson, Short Range Resonating Valence Bond Theories and Superconductivity, Mod. Phys. Lett. B 04, 225 (1990).

[66] L. Balents, Spin Liquids in Frustrated Magnets, Nature (London) 464, 199 (2010).

[67] N. Read and S. Sachdev, Valence-Bond and Spin-Peierls Ground States of Low-Dimensional Quantum Antiferromagnets, Phys. Rev. Lett. 62, 1694 (1989).

[68] N. Read and S. Sachdev, Spin-Peierls, Valence-Bond Solid, and Néel Ground States of Low-Dimensional Quantum Antiferromagnets, Phys. Rev. B 42, 4568 (1990).

[69] T. Senthil, A. Vishwanath, L. Balents, S. Sachdev, and M. P. A. Fisher, Deconfined Quantum Critical Points, Science 303, 1490 (2004).
[70] D. Banerjee, F. J. Jiang, P. Widmer, and U. J. Wiese, The $(2+1)-d U(1)$ Quantum Link Model Masquerading as Deconfined Criticality, J. Stat. Mech. 12, P12010 (2013).

[71] J. Villian, R. Bidaux, J. P. Carton, and R. Conte, Order as an Effect of Disorder, J. Phys. (Paris) 41, 1263 (1980).

[72] R. A. Jalabert and S. Sachdev, Spontaneous Alignment of Frustrated Bonds in an Anisotropic, Three-Dimensional Ising Model, Phys. Rev. B 44, 686 (1991).

[73] Charge conjugation implies that the electric field and, thus, the link spins are reversed.

[74] Contrary to what occurs for the square lattice, such Hamiltonians cannot be simply mapped into U(1) gauge theories of the form (4) on the corresponding lattices.

[75] R. Moessner and S. L. Sondhi, Resonating Valence Bond Phase in the Triangular Lattice Quantum Dimer Model, Phys. Rev. Lett. 86, 1881 (2001).

[76] N. Shannon, G. Misguich, and K. Penc, Cyclic Exchange, Isolated States, and Spinon Deconfinement in an XXZ Heisenberg Model on the Checkerboard Lattice, Phys. Rev. B 69, 220403(R) (2004).

[77] G. Misguich and F. Mila, Quantum Dimer Model on the Triangular Lattice: Semiclassical and Variational Approaches to Vison Dispersion and Condensation, Phys. Rev. B 77, 134421 (2008).

[78] H. P. Büchler, M. Hermele, S. D. Huber, M. P. A. Fisher, and P. Zoller, Atomic Quantum Simulator for Lattice Gauge Theories and Ring Exchange Models, Phys. Rev. Lett. 95, 040402 (2005).

[79] H. Weimer, M. Müller, I. Lesanovsky, P. Zoller, and H. P. Büchler, A Rydberg Quantum Simulator, Nat. Phys. 6, 382 (2010).

[80] M. Müller, I. Lesanovsky, H. Weimer, H. P. Büchler, and P. Zoller, Mesoscopic Rydberg Gate Based on Electromagnetically Induced Transparency, Phys. Rev. Lett. 102, 170502 (2009).

[81] W. Zheng and S. Sachdev, Sine-Gordon Theory of the NonNéel Phase of Two-Dimensional Quantum Antiferromagnets, Phys. Rev. B 40, 2704(R) (1989).

[82] L. S. Levitov, Equivalence of the Dimer ResonatingValence-Bond Problem to the Quantum Roughening Problem, Phys. Rev. Lett. 64, 92 (1990).

[83] C. L. Henley, Relaxation Time for a Dimer Covering with Height Representation, J. Stat. Phys. 89, 483 (1997).

[84] A. Celi et al. (to be published).

[85] The duality introduced here is different than the one first noticed in Ref. [86] between the dimer models on the square and hexagonal lattices and fully frustrated Ising model on their dual lattices. In fact, the realization of the generalized blockade Hamiltonian in terms of Rydberg atoms in Sec. III is equivalent to the realization of an anisotropic Ising model with first and second neighbor couplings on properly tuned geometries.

[86] R. Moessner, S. L. Sondhi, and P. Chandra, TwoDimensional Periodic Frustrated Ising Models in a Transverse Field, Phys. Rev. Lett. 84, 4457 (2000).

[87] ITensor library (version 2.3), http://itensor.org.

[88] P. Fendley, K. Sengupta, and S. Sachdev, Competing Density-Wave Orders in a One-Dimensional Hard-Boson Model, Phys. Rev. B 69, 075106 (2004). 
[89] R. Samajdar, S. Choi, H. Pichler, M. D. Lukin, and S. Sachdev, Numerical Study of the Chiral $Z_{3}$ Quantum Phase Transition in One Spatial Dimension, Phys. Rev. A 98, 023614 (2018).

[90] R. Moessner and S. L. Sondhi, Ising Models of Quantum Frustration, Phys. Rev. B 63, 224401 (2001).

[91] J. Herzog-Arbeitman, S. Mantilla, and I. Sodemann, Solving the Quantum Dimer and Six Vertex Models One Electric Field Line at a Time, Phys. Rev. B 99, 245108 (2019).

[92] J. Zhang, J. Unmuth-Yockey, J. Zeiher, A. Bazavov, S.-W. Tsai, and Y. Meurice, Quantum Simulation of the Universal Features of the Polyakov Loop, Phys. Rev. Lett. 121, 223201 (2018).

[93] E. Zohar and J. I. Cirac, Removing Staggered Fermionic Matter in $U(N)$ and $S U(N)$ Lattice Gauge Theories, Phys. Rev. D 99, 114511 (2019).

[94] R. Moessner and S. L. Sondhi, Three-Dimensional Resonating-Valence-Bond Liquids and Their Excitations, Phys. Rev. B 68, 184512 (2003).

[95] M. Hermele, M. P. A. Fisher, and L. Balents, Pyrochlore Photons: The U(1) Spin Liquid in a $S=\frac{1}{2}$ ThreeDimensional Frustrated Magnet, Phys. Rev. B 69, 064404 (2004).

[96] E. Fradkin, D. A. Huse, R. Moessner, V. Oganesyan, and S. L. Sondhi, Bipartite Rokhsar-Kivelson Points and Cantor Deconfinement, Phys. Rev. B 69, 224415 (2004).

[97] A. Vishwanath, L. Balents, and T. Senthil, Quantum Criticality and Deconfinement in Phase Transitions between Valence Bond Solids, Phys. Rev. B 69, 224416 (2004).

[98] S. Papanikolaou, K. S. Raman, and E. Fradkin, Devil's Staircases, Quantum Dimer Models, and Stripe Formation in Strong Coupling Models of Quantum Frustration, Phys. Rev. B 75, 094406 (2007).

[99] C. Castelnovo, R. Moessner, and S. L. Sondhi, Magnetic Monopoles in Spin Ice, Nature (London) 451, 42 (2008).

[100] R. Islam, R. Ma, P. M. Preiss, M. E. Tai, A. Lukin, M. Rispoli, and M. Greiner, Measuring Entanglement Entropy in a Quantum Many-Body System, Nature (London) 528, 77 (2015).

[101] T. Brydges, A. Elben, P. Jurcevic, B. Vermersch, C. Maier, B. P. Lanyon, P. Zoller, R. Blatt, and C. F. Roos, Probing Rényi Entanglement Entropy via Randomized Measurements, Science 364, 260 (2019).

[102] C. M. Alves and D. Jaksch, Multipartite Entanglement Detection in Bosons, Phys. Rev. Lett. 93, 110501 (2004).

[103] A. J. Daley, H. Pichler, J. Schachenmayer, and P. Zoller, Measuring Entanglement Growth in Quench Dynamics of Bosons in an Optical Lattice, Phys. Rev. Lett. 109, 020505 (2012).

[104] D. A. Abanin and E. Demler, Measuring Entanglement Entropy of a Generic Many-Body System with a Quantum Switch, Phys. Rev. Lett. 109, 020504 (2012).

[105] A. Elben, B. Vermersch, M. Dalmonte, J. I. Cirac, and P. Zoller, Rényi Entropies from Random Quenches in Atomic Hubbard and Spin Models, Phys. Rev. Lett. 120, 050406 (2018).
[106] G. Torlai, G. Mazzola, J. Carrasquilla, M. Troyer, R. Melko, and G. Carleo, Neural-Network Quantum State Tomography, Nat. Phys. 14, 447 (2018).

[107] G. Torlai et al., Integrating Neural Networks with a Quantum Simulator for State Reconstruction, Phys. Rev. Lett. 123, 230504 (2019).

[108] I. Cong, S. Choi, and M. D. Lukin, Quantum Convolutional Neural Networks, Nat. Phys. 15, 1273 (2019).

[109] F. Tschirsich, S. Montangero, and M. Dalmonte, Phase Diagram and Conformal String Excitations of Square Ice Using Gauge Invariant Tensor Networks, SciPost Phys. 6, 028 (2019).

[110] A. Keesling et al., Quantum Kibble-Zurek Mechanism and Critical Dynamics on a Programmable Rydberg Simulator, Nature (London) 568, 207 (2019).

[111] C. J. Turner, A. A. Michailidis, D. A. Abanin, M. Serbyn, and Z. Papic, Weak Ergodicity Breaking from Quantum Many-Body Scars, Nat. Phys. 14, 745 (2018).

[112] W. W. Ho, S. Choi, H. Pichler, and M. D. Lukin, Periodic Orbits, Entanglement, and Quantum Many-Body Scars in Constrained Models: Matrix Product State Approach, Phys. Rev. Lett. 122, 040603 (2019).

[113] T. V. Zache, N. Mueller, J. T. Schneider, F. Jendrzejewski, J. Berges, and P. Hauke, Dynamical Topological Transitions in the Massive Schwinger Model with a $\theta$ Term, Phys. Rev. Lett. 122, 050403 (2019).

[114] Y.-P. Huang, D. Banerjee, and M. Heyl, Dynamical Quantum Phase Transitions in U(1) Quantum Link Models, Phys. Rev. Lett. 122, 250401 (2019).

[115] Z. Lan, M. van Horssen, S. Powell, and J. P. Garrahan, Quantum Slow Relaxation and Metastability due to Dynamical Constraints, Phys. Rev. Lett. 121, 040603 (2018).

[116] J. Feldmeier, F. Pollmann, and M. Knap, Emergent Glassy Dynamics in a Quantum Dimer Model, Phys. Rev. Lett. 123, 040601 (2019).

[117] For an example of quantum cellular automata, see, e.g., Ref. [118]; for a precise definition, see Refs. [119,120], and references therein.

[118] D. Bleh, T. Calarco, and S. Montangero, Quantum Game of Life, Europhys. Lett. 97, 20012 (2012).

[119] P. Arrighi, An Overview of Quantum Cellular Automata, arXiv:1904.12956.

[120] T. Farrelly, A Review of Quantum Cellular Automata, arXiv:1904.13318.

[121] H. Pichler, S.-T. Wang, L. Zhou, S. Choi, and M. D. Lukin, Quantum Optimization for Maximum Independent Set Using Rydberg Atom Arrays, arXiv:1808.10816.

[122] A. Cooper, J. P. Covey, I. S. Madjarov, S. G. Porsev, M. S. Safronova, and M. Endres, Alkaline-Earth Atoms in Optical Tweezers, Phys. Rev. X 8, 041055 (2018).

[123] M. A. Norcia, A. W. Young, and A. M. Kaufman, Microscopic Control and Detection of Ultracold Strontium in Optical-Tweezer Arrays, Phys. Rev. X 8, 041054 (2018).

[124] S. Saskin, J. Wilson, B. Grinkemeyer, and J. Thompson, Narrow-Line Cooling and Imaging of Ytterbium Atoms in an Optical Tweezer Array, Phys. Rev. Lett. 122, 143002 (2019). 